University of Nebraska - Lincoln

DigitalCommons@University of Nebraska - Lincoln

October 1999

\title{
High-order harmonic generation in magnetic and parallel magnetic and electric fields
}

\author{
Dejan B. Miloševic \\ University of Nebraska - Lincoln \\ Anthony F. Starace \\ University of Nebraska-Lincoln, astarace1@unl.edu
}

Follow this and additional works at: https://digitalcommons.unl.edu/physicsstarace

Part of the Physics Commons

Miloševic, Dejan B. and Starace, Anthony F., "High-order harmonic generation in magnetic and parallel magnetic and electric fields" (1999). Anthony F. Starace Publications. 72.

https://digitalcommons.unl.edu/physicsstarace/72

This Article is brought to you for free and open access by the Research Papers in Physics and Astronomy at DigitalCommons@University of Nebraska - Lincoln. It has been accepted for inclusion in Anthony F. Starace Publications by an authorized administrator of DigitalCommons@University of Nebraska - Lincoln. 


\title{
High-order harmonic generation in magnetic and parallel magnetic and electric fields
}

\author{
Dejan B. Milošević* and Anthony F. Starace \\ Department of Physics and Astronomy, The University of Nebraska, 116 Brace Laboratory, Lincoln, Nebraska 68588-0111
}

(Received 1 April 1999)

\begin{abstract}
We demonstrate control of high-harmonic generation (HHG) by a linearly polarized laser field using uniform static magnetic and electric fields parallel to the laser polarization. We show that the harmonic intensity can be considerably increased for particular values of the magnetic field. The maximum values of the harmonic intensity correspond to such values of magnetic induction for which an integer multiple of the classical cyclotron period of the electron's motion perpendicular to the magnetic field is equal to the return time of the ionized electron wave packet to the nucleus under the influence of the laser field (and static electric field, if present). While a static magnetic field (for the strengths we are considering) only affects the cutoff position slightly, a static electric field can introduce additional plateaus and cutoffs. A properly chosen combination of the static electric and magnetic fields can increase both the harmonic intensity and the harmonic order. For the case of a magnetic field only, the present work expands upon a brief account recently given elsewhere [D. B. Milošević and A. F. Starace, Phys. Rev. Lett. 82, 2653 (1999)]. For both a static B field and for parallel static $B$ and $E$ fields, the present work provides further confirmation of the so called "three-step" model for interpreting quantum-mechanical predictions of HHG. [S1050-2947(99)06110-7]

PACS number(s): 32.80.Qk, 42.65.Ky, 42.50.Hz, 32.80.Wr
\end{abstract}

\section{INTRODUCTION}

Atomic processes in the presence of strong fields are presently attracting considerable attention, as indicated by numerous books [1-3] and conference proceedings [4-7], as well as by recent review articles devoted to a number of particular areas of multiphoton physics, such as ionization dynamics in strong laser fields [8], two- and three-step models for intense-field, laser-atom physics [9], atomic physics with high-intensity lasers [10], $R$-matrix-Floquet theory of multiphoton processes [11], laser-assisted electron-atom scattering [12], and two-electron atoms in strong fields [13]. In the present paper we consider another process requiring a strong laser field: high-harmonic generation (HHG) [4-10,14-16]. The main features of this process, namely an extended plateau, comprising many harmonics with comparable intensities, and a sharp, high-frequency cutoff, are explained using a "three-step" physical model [17,18] (for a review see, e.g., Ref. [9]). According to this model, the "first step" is ionization, the "second step" is laser-driven propagation of the free electron, and the "third step" is the collision of the electron (or, more precisely, its wave packet) with the atomic core under the driving influence of the laser field. During this collision the electron can recombine with the core and emit a harmonic photon. This model predicts the maximum energy $\left(N_{\max } \hbar \omega\right)$ of the harmonics at the cutoff to be equal to $I_{p}+3.17 U_{p}$, where $I_{p}$ is the atomic ionization potential and $U_{p}$ is the ponderomotive energy, $U_{p}$ $=e^{2} E_{L}^{2} /\left(4 m_{e} \omega^{2}\right)$, where $-e$ and $m_{e}$ are the electron charge and mass, and $E_{L}$ and $\omega$ are the laser electric field amplitude and frequency, respectively. Besides the position of the cut-

\footnotetext{
* On leave from: Faculty of Science and Mathematics, Department of Physics, University of Sarajevo, Zmaja od Bosne 35, 71000 Sarajevo, Bosnia and Herzegovina. Present address: Max-BornInstitut, Max-Born-Str. 2A, 12489 Berlin, Germany.
}

off, another important characteristic of the HHG process is its efficiency, which is determined by the intensities of the harmonics on the plateau.

Many efforts have been made to control HHG. This control can be achieved by adding a second component to the laser field or by adding a static electric or magnetic field. The analyses of HHG in a bichromatic laser field (Refs. [19,20], and references therein) have shown that such control is possible: in both the $(\omega, 3 \omega)$ and the $(\omega, 2 \omega)$ cases the efficiency of odd harmonic generation can be increased by orders of magnitude, while in the $(\omega, 2 \omega)$ case the generation of even harmonics, in addition to odd ones, is possible. In both cases there is an additional parameter - the relative phase between the laser field components - which enables coherent phase control. Even for a monochromatic laser field, in the case of ultrashort laser field pulses, the initial laser field phase has a significant influence on the HHG process [21]. HHG also strongly depends on the polarization of the laser field $[20,22]$. The best efficiency can be achieved with linearly polarized laser fields. Recently, HHG from an initial, coherent superposition of atomic states has been explored theoretically [23]. Addition of a static electric field to control the HHG process has also been explored theoretically [2426]. In this latter case even harmonics can be generated and, more important, it is possible to generate harmonics beyond the cutoff in the absence of the static field $[25,26]$. Coherent control of HHG by a static magnetic field was proposed by Bandrauk and co-workers [27-29]. They have considered two cases: (i) the $\mathrm{H}_{2}^{+}$molecular ion in both a linearly polarized laser field and an ultrastrong, static magnetic field along the laser polarization axis and (ii) a two-dimensional model of the hydrogen atom in both a circularly polarized laser field and a magnetic field perpendicular to the laser field polarization plane. In Refs. [27-29] results are presented for only one value of the magnetic induction, $B=0.2 B_{0}=47000 \mathrm{~T}$, where $B_{0}=\hbar /\left(e a_{0}^{2}\right)=2.3505 \times 10^{5} \mathrm{~T}$. This value of $B$ is much larger than the maximum presently achievable laboratory magnetic field (see below). Connerade and Keitel [30] 
also investigated theoretically HHG in a static magnetic field. They considered relativistically strong laser fields and concentrated on the influence of a strong constant magnetic field on free electrons in such laser fields using a classical treatment. A similar problem was considered more recently by Salamin and Faisal [31]. They presented exact analytic solutions for fully relativistic electron trajectories in the presence of a superintense laser field and a strong uniform magnetic field. Using these solutions they analyzed the light emission spectrum along various directions of observation and as functions of the intensity and frequency of the laser as well as the strength of the magnetic field.

The study of atomic processes in a magnetic field is a long established area of atomic physics, dating from old discoveries by Zeeman and Lorentz [32]. Under normal laboratory conditions the energy changes caused by the magnetic interaction are usually small compared with the characteristic energies of the system. However, in experiments with highly excited atoms (in which the bound-state energy $E_{n}$ is small for large $n$ ), in solid state physics (in which the effective mass is much smaller than the electron mass $m_{e}$ and the dielectric constant can be much larger than 1), and in astrophysics (where the magnetic induction in white dwarfs, pulsars, and neutron stars can reach $10^{3}-10^{8} \mathrm{~T}$ ) this energy change can be important. For reviews see, for example, Refs. [32-36]. The maximum reproducible laboratory magnetic fields which have been reported have an induction $B$ $\approx 1000 \mathrm{~T}$ [37]. In the experiment presented in Ref. [37] the useful volume having this maximum magnetic field consists of a cylinder approximately $1 \mathrm{~cm}$ in diameter and $10 \mathrm{~cm}$ long. The duration of such strong magnetic pulses is a few $\mu \mathrm{s}$, which is much larger than the laser field pulse duration, so that we can consider the magnetic field as constant. With the recent development of ultrastrong laser pulses (with intensities of order $10^{20} \mathrm{~W} / \mathrm{cm}^{2}$, developed in connection with such applications as a fast ignition scheme for inertial confinement fusion) it becomes possible to generate magnetic fields up to $10^{4} \mathrm{~T}$ by propagation of relativistically intense laser pulses through preionized plasmas [38]. (See also Ref. [39] concerning magnetic fields generated in a plasma by a short, circularly polarized laser pulse.)

In this paper we demonstrate control of HHG using a strong, uniform static magnetic field directed along the polarization axis of a linearly polarized laser field. We explain here also the physical mechanism which enables this control and the optimal experimental parameters for maximizing the intensities of high harmonics. Our presentation here expands upon a brief account we have recently given elsewhere [40]. In particular, in Ref. [40] we have shown that maxima in the intensities of harmonics generated in a strong magnetic field may be understood on the basis of the classical "three-step" model $[9,17,18]$, i.e., by considering the Newtonian trajectories of an intermediate-state electron moving under the influence of the laser field along the $z$ axis. Because for experimentally obtainable fields one has $B \ll B_{0}$, the magnetic field's influence on the atomic ground state can be neglected [41]. In this approximation the "first" and the "third step" of the above-mentioned "three-step" model are not affected by the magnetic field. Therefore, the main influence of the magnetic field is on the "second step," i.e., on the electron's propagation, which mainly determines the harmonic spec- trum. One of the reasons for the decrease of harmonic efficiency is the spreading (in the transverse direction) of the electron wave packet during the propagation in the linearly polarized laser field. The addition of the magnetic field along the laser field polarization axis (chosen in the $z$ direction) suppresses this spreading. It acts as a transverse parabolic barrier in the $\rho$ direction [see the term proportional to $\rho^{2}$ $=x^{2}+y^{2}$ in Eq. (A7)], which can be considered as a magnetic bottle confining the ionized electron wave packet transverse to the magnetic field axis. Therefore, we expect that the addition of a strong magnetic field can increase the efficiency of HHG. We demonstrate that maxima in the intensities of the generated harmonics occur when the classical periods for electronic motion perpendicular and parallel to the $z$ axis have a rational ratio. The case of HHG in parallel static $E$ and $B$ fields provides a more stringent test of the utility of the "three-step" model for interpreting the results of quantum mechanical calculations. For the case of a static $B$ field alone, the classical time required for the intermediate-state electron to return to the nucleus, whereupon it may rescatter from the atomic core and emit a harmonic photon, is invariant to a change of phase of $\pi$ of the driving laser field. For the case of parallel static $E$ and $B$ fields, however, the periods of the classical trajectories which return to the origin are sensitive to the initial orientation of the laser polarization with respect to the static electric field. We find, remarkably, a one-to-one correspondence between detailed features of our quantummechanically calculated harmonic intensities and the variety of classical trajectories that occur in the parallel static fields case. These results, presented below, thus represent a further confirmation of the validity of the "three-step" classical model.

Besides the increase of the HHG efficiency, the cutoff energy in the magnetic field is increased by the Landau energy $E_{n m}$ [42] [See also Eq. (A8).] Because this energy is proportional to $B / B_{0}$, the cutoff energy remains almost unchanged for the field values we are considering. Contrary to this, the addition of a static electric field can change considerably the cutoff energy, and can even introduce additional plateaus with their own cutoffs [24-26]. This is because a parallel static electric field comes into the stationary action on the same footing as the laser field [see Eqs. (5) and (A6) below], and also gives a term proportional to $E_{S}^{2} \tau^{3}$, which can be large for long return times $\tau$ [43]. We thus find that in parallel static $B$ and $E$ fields, the static magnetic field increases the HHG efficiency and the static electric field increases the cutoff energy.

In Sec. II we present briefly the theory of HHG in the presence of static fields. Details of the theory presented in this section are relegated to the Appendices. Our numerical results are presented in Sec. III, while in Sec. IV we interpret these results using a classical analysis. Section V presents our conclusions.

\section{THEORY}

We obtain harmonic spectra by computing the quantummechanical dipole moment $\mathbf{D}_{N}$, which is defined as the Fourier transform of the time-dependent dipole matrix element $\mathbf{d}(t)=\langle\Phi(t)|e \mathbf{r}| \Phi(t)\rangle$, where $|\Phi(t)\rangle$ is the solution of the Schrödinger equation for the system (atom + laser field + 
static fields). Using the strong-field approximation and neglecting continuum-continuum coupling, the time-dependent dipole can be written as [15]

$$
\mathbf{d}(t)=\int_{t_{0}}^{t} d t^{\prime}\left\langle u(t)\left|e \mathbf{r} G_{L}\left(t, t^{\prime}\right) e \mathbf{E}_{L}\left(t^{\prime}\right) \cdot \mathbf{r}\right| u\left(t^{\prime}\right)\right\rangle+\text { c.c. }
$$

where $G_{L}\left(t, t^{\prime}\right)$ is the Volkov-type Green's operator for the electron in the presence of the laser field and the static fields, and $e \mathbf{E}_{L}\left(t^{\prime}\right) \cdot \mathbf{r}$ is the interaction of the electron with the laser field in the length gauge (see Appendix A). We assume that the static fields are not strong enough to modify the atomic ground state $|u(t)\rangle=\left|u_{0}\right\rangle \exp \left(i I_{p} t / \hbar\right)$. However, they are included in $G_{L}\left(t, t^{\prime}\right)$ and thus have an influence on the electron's intermediate states. In the case of parallel linearly polarized fields we have to compute the dipole moment

$$
D_{N}=\int_{0}^{T} \frac{d t}{T} d(t) \exp (i N \omega t)
$$

where the laser field period $T$ equals $2 \pi / \omega$ and where, according to Eq. (1) and the results of Appendix A,

$$
\begin{aligned}
d(t)= & \frac{-i e^{2}}{\hbar} \int_{-\infty}^{t} d t^{\prime} E_{L}\left(t^{\prime}\right) \int_{-\infty}^{\infty} d q \\
& \times \sum_{n, m}\left\langle u_{0}|z| n m Q(t)\right\rangle\left\langle n m Q\left(t^{\prime}\right)|z| u_{0}\right\rangle \\
& \times \exp \left[-\frac{i}{\hbar} S_{n m}\left(q ; t, t^{\prime}\right)\right]+\text { c.c. }
\end{aligned}
$$

where

$$
\begin{aligned}
& Q(t)=q+\frac{e}{\hbar}\left[A_{L}(t)+A_{S}(t)\right] \\
& S_{n m}\left(q ; t, t^{\prime}\right)= \int_{t^{\prime}}^{t} d t^{\prime \prime} \frac{\hbar^{2}}{2 m_{e}}\left\{q+\frac{e}{\hbar}\left[A_{L}\left(t^{\prime \prime}\right)+A_{S}\left(t^{\prime \prime}\right)\right]\right\}^{2} \\
&+\left(I_{p}+E_{n m}\right)\left(t-t^{\prime}\right) \\
&= \hbar q\left[\alpha(t)-\alpha\left(t^{\prime}\right)\right]+U(t)-U\left(t^{\prime}\right) \\
&+\left(\frac{\hbar^{2} q^{2}}{2 m_{e}}+I_{p}+E_{n m}\right)\left(t-t^{\prime}\right),
\end{aligned}
$$

and $\alpha(t), U(t)$ and $E_{n m}$ are defined in Appendix A. The method of computation of the matrix elements with the wavefunctions $\langle\rho \phi z \mid n m q\rangle \equiv \Psi_{n m q}(\rho, \phi, z, 0)$ is given in the appendices. The integral over the $z$-component of the intermediate electron momentum $\hbar q$ is computed using the saddle-point method, as in Refs. [15,20]. This method gives the factor $\left[2 \pi m_{e} \hbar /(i \tau)\right]^{1 / 2} \exp \left[-i S_{s}(t, \tau) / \hbar\right]$, where $S_{s}(t, \tau)$ $\equiv S_{n m}\left(q_{s} ; t, t-\tau\right)$ is the stationary action, and $\hbar q_{s}$ is the stationary momentum

$$
\begin{aligned}
\hbar q_{s}(t, \tau) & =-\frac{e}{\tau} \int_{t-\tau}^{t} d t^{\prime \prime}\left[A_{L}\left(t^{\prime \prime}\right)+A_{S}\left(t^{\prime \prime}\right)\right] \\
& =\frac{m_{e}}{\tau}[\alpha(t-\tau)-\alpha(t)], \quad \tau=t-t^{\prime}
\end{aligned}
$$

The final result for the time-dependent dipole moment is

$$
\begin{aligned}
d(t)= & -i e^{2}\left(\frac{2 \pi m_{e}}{i \hbar^{3}}\right)^{1 / 2} \int_{0}^{\infty} \frac{d \tau}{\tau^{1 / 2}} E_{L}(t-\tau) \\
& \times \sum_{n, m}\left\langle u_{0}|z| n m Q_{s}(t)\right\rangle\left\langle n m Q_{s}(t-\tau)|z| u_{0}\right\rangle \\
& \times \exp \left[-\frac{i}{\hbar} S_{s}(t, \tau)\right]+\text { c.c. }
\end{aligned}
$$

An analysis of this result is presented in the appendices. The summation over the quantum numbers $n$ and $m$ can be performed analytically; the result for the summed product of the matrix elements in Eq. (7) is a single integral over exponential integral functions. This integral can be efficiently computed by the method described in Appendix C. It should be mentioned that in the absence of the magnetic field one must compute the time-dependent dipole in a different way. Instead of the summation over the quantum numbers $n$ and $m$ one has infinite integrals over the $x$ and $y$ components of the intermediate electron momentum (i.e., $q_{x}$ and $q_{y}$ ). One can evaluate these integrals by similarly applying the saddlepoint method, as was done with the integration over the $z$ component. As a result one obtains a term with the factor $\tau^{-3 / 2}$, instead of $\tau^{-1 / 2}$, in Eq. (7), and the integrand of Eq. (7) has a simple analytic form $[15,20]$. This simplicity is important because the integration over $\tau$ in Eq. (7) can cause problems in the case of only a static electric field. (The upper limit of the integral over $\tau$ should be large because in the presence of a static electric field the terms with large return time can give significant contributions.)

\section{RESULTS}

\section{A. Harmonic intensity revivals in a magnetic field}

We first present our results for the case of a magnetic field only. In Fig. 1 we present the harmonic intensities (i.e., the harmonic generation efficiencies, defined as $\left|D_{N}\right|^{2}$ ) as functions of the harmonic order $N$ for different values of the magnetic induction: $B=0 \mathrm{~T}$ (squares), $2000 \mathrm{~T}$ (circles), and $4000 \mathrm{~T}$ (triangles). The laser field intensity and photon energy are $I=5 \times 10^{10} \mathrm{~W} / \mathrm{cm}^{2}$ and $\hbar \omega=0.1165 \mathrm{eV}\left(\mathrm{CO}_{2}\right.$ laser), respectively. For the $\mathrm{H}^{-}$ion $I_{p}=0.754 \mathrm{eV}$ and the ground state wave function has the form of Eq. (B4) with $a=0$ and $b=1$. We observe immediately that in general the harmonic intensities for $N \geqslant 5$ are one or more orders of magnitude greater in the presence of the $B$ field than for the case $B=0$, although there are exceptions (e.g., $N=9$ or 21 ). Only the odd harmonics are generated because the parallel magnetic field preserves axial symmetry. The cutoff of the plateau for $B=0$ T should be (according to the "three-step" model) at $3.17 U_{p}+I_{p}=14.4 \hbar \omega+6.5 \hbar \omega=20.9 \hbar \omega$, so that $N_{\text {max }} \approx 21$, which agrees with the results presented in Fig. 1 . 


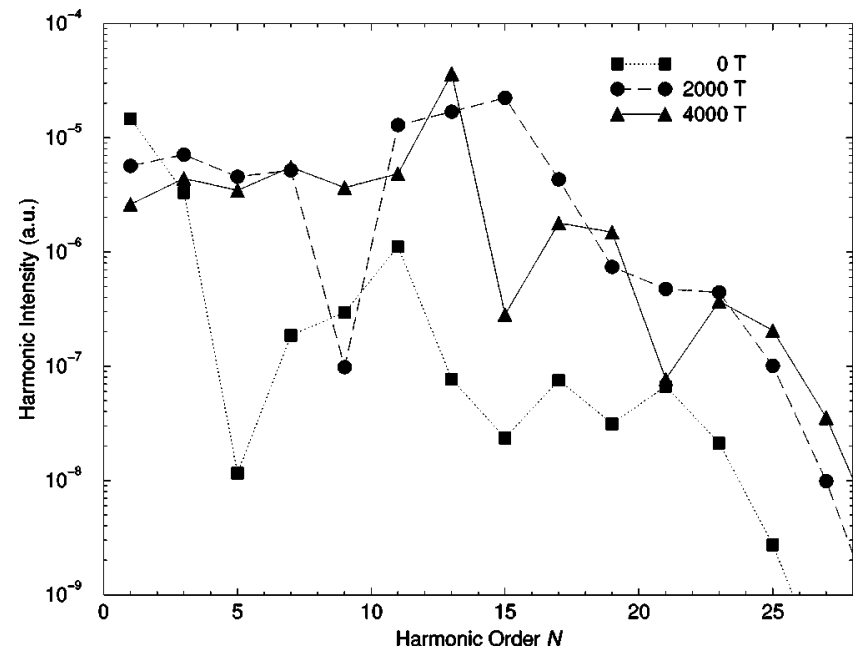

FIG. 1. Harmonic intensities as functions of the harmonic order $N$ for the $\mathrm{H}^{-}$ion in a $\mathrm{CO}_{2}$ laser with the intensity $I=5$ $\times 10^{10} \mathrm{~W} / \mathrm{cm}^{2}$. The magnetic field induction is: $B=0 \mathrm{~T}$ (squares), $2000 \mathrm{~T}$ (circles), and $4000 \mathrm{~T}$ (triangles).

In the presence of the magnetic field the cutoff energies are increased by the ground-state Landau level energy $\hbar \omega_{B} / 2$ $=e \hbar B /\left(2 m_{e}\right)$, which, for $B=4000 \mathrm{~T}$ and a $\mathrm{CO}_{2}$ laser, is $\hbar \omega_{B} / 2=1.987 \hbar \omega$, giving a plateau cutoff of $\approx 22.9 \hbar \omega$. For $B=2000 \mathrm{~T}$ the maximum harmonic intensity on the plateau occurs for $N=15$, while for $B=4000$ T the maximum occurs for $N=13$. One can observe also sharp variations of intensity as $B$ is varied for particular harmonics (e.g., $N=9,15$, and $21)$. In order to explore the dependence of the harmonic intensity on the strength of the magnetic field, in Fig. 2 we present a 3D plot of the harmonic intensities (expressed in $10^{-5}$ a.u. on a linear scale) as functions of the harmonic order $N$ and the magnetic induction $B$. The pronounced maxima for particular values of $B$ are more clearly visible in Fig. 2. For fixed $N$, one can also notice a periodicity of the

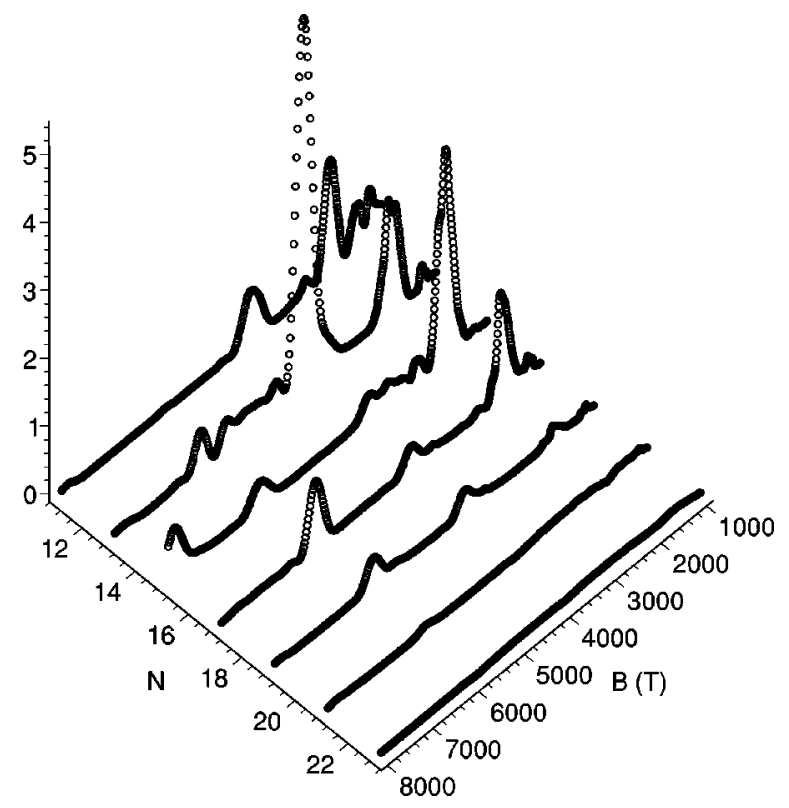

FIG. 2. Harmonic intensities (in units of $10^{-5}$ a.u.) as functions of the harmonic order $N$ and the magnetic field induction $B$. The laser field and the $\mathrm{H}^{-}$ion parameters are as in Fig. 1.

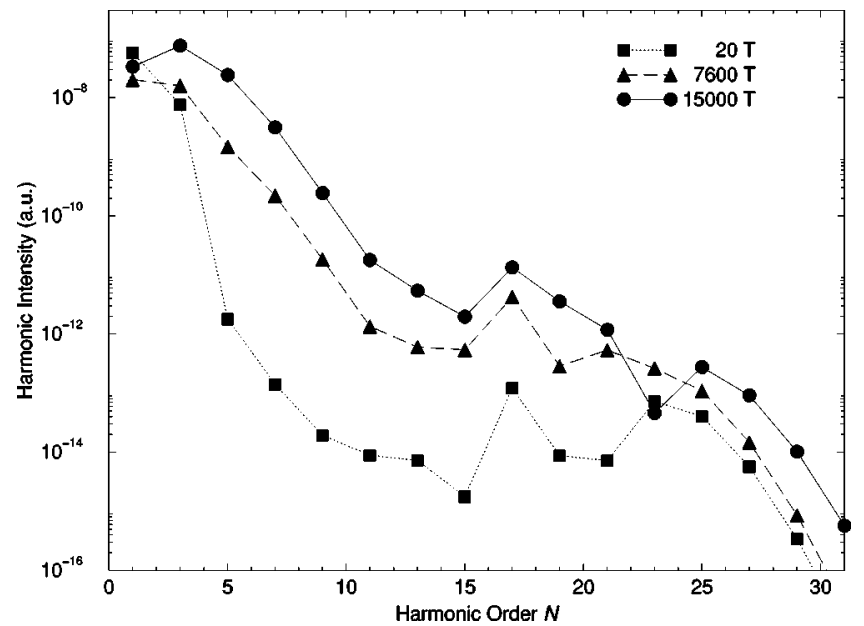

FIG. 3. The same as in Fig. 1, but for argon atoms in the presence of a Nd:YAG laser having intensity $I=3 \times 10^{13} \mathrm{~W} / \mathrm{cm}^{2}$. Results for three values of the magnetic field induction are shown: $B$ $=20 \mathrm{~T}$ (squares), $7600 \mathrm{~T}$ (triangles), and $15000 \mathrm{~T}$ (circles).

appearance of these maxima at $B \approx 2000,4000,6000,8000$ $\mathrm{T}, \ldots$ We will refer to this periodicity as revivals of the harmonic intensity. There thus appears to be an optimum value of the magnetic induction for which the harmonic intensity of a fixed harmonic has a maximum. In the next section we connect these values with particular classical electron trajectories.

In order to test the "three-step" model for a very different set of parameters, we have also carried out quantummechanical calculations of HHG for argon atoms [having $I_{p}=15.76 \mathrm{eV}$, and using a hydrogenic ground state wave function given by Eq. (B4) with $a=1$ and $b=0$ ] in the presence of a Nd:YAG laser ( $\hbar \omega=1.165 \mathrm{eV}$ ) having intensity $I=3 \times 10^{13} \mathrm{~W} / \mathrm{cm}^{2}$. In this case the cutoff for $B=0$ is at $I_{p}$ $+3.17 U_{p}=22.2 \hbar \omega$ and $I_{p}, I$, and $\omega$ are all much larger. Hence in order to have a cyclotron period comparable to the laser field period, $B$ must be increased. (Similarly, if we were to consider a laser having a much smaller frequency $\omega$, then much smaller values of the static magnetic field $B$ are needed in order that the cyclotron period is comparable to the classical period of motion of the electron under the driving influence of the laser field.) In Fig. 3 we present the harmonic intensities as functions of the harmonic order for $B=20$, 7600, and $15000 \mathrm{~T}$. In general one sees that the higher the value of $B$, the higher is the harmonic intensity. Indeed, for some values of $B$ the harmonic intensity is increased by almost four orders of magnitude. In Fig. 4 we present the harmonic intensities for the 13th, 15th, 17th, and 19th harmonics as functions of the magnetic induction $B$. The harmonic intensities have maxima for values of $B$ close to $13000 \mathrm{~T}$, with the largest intensities for these four harmonics occuring in the range $10000 \mathrm{~T} \leqslant B \leqslant 16000 \mathrm{~T}$. Within this region the harmonic intensities are many orders of magnitude greater than for $B=0$.

\section{B. Additional plateaus and cutoffs in parallel static electric and magnetic fields}

In Fig. 5 we present $\mathrm{HHG}$ results for the $\mathrm{H}^{-}$ion for the case of a $\mathrm{CO}_{2}$ laser and parallel static electric and magnetic 


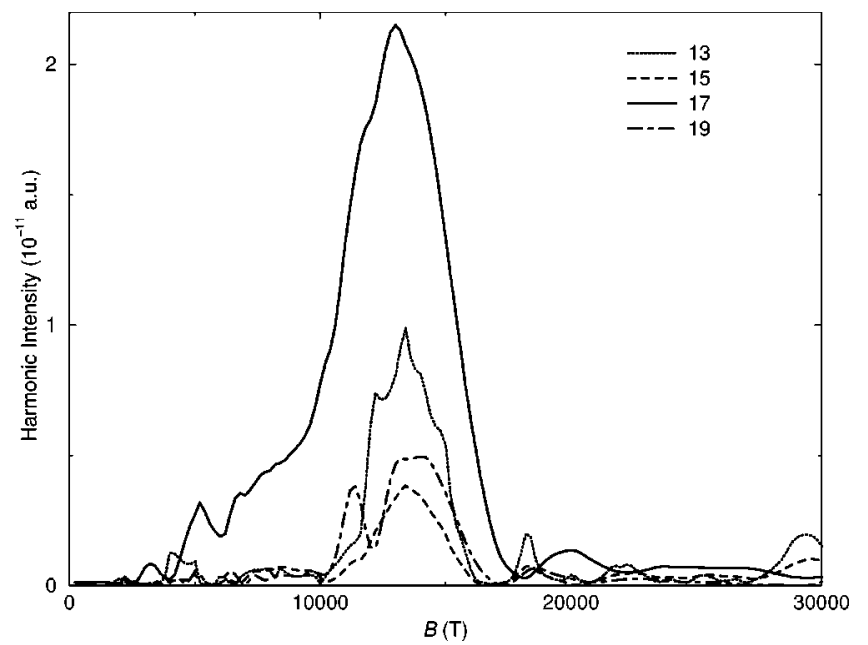

FIG. 4. Harmonic intensities as functions of the magnetic field induction $B$ for fixed harmonic order: $N=13$ (dotted line), 15 (dashed line), 17 (solid line), and 19 (dot-dashed line). The atomic and the laser field parameters are as in Fig. 3.

fields. For numerical convenience, we have selected a static electric field having strength $E_{S}=1 \mathrm{MV} / \mathrm{cm}$. Such strong static fields can be achieved in experiments using relativistic $\mathrm{H}^{-}$atom beams to convert a modest transverse laboratory magnetic field into a static electric field in the atom's rest frame [44]. However, such an approach is not appropriate if one wants to have simultaneously a strong parallel magnetic field. Currently static fields of the order of $0.1 \mathrm{MV} / \mathrm{cm}$ have been achieved in the design of particle accelerators [45]. However, since our primary purpose in considering the case of HHG in parallel static $E$ and $B$ fields is to provide a more stringent test of the "three-step" model of HHG, the stronger the fields, the more clear are the theoretical results. Nevertheless, we expect our theoretical predictions to apply qualitatively to experiments involving smaller values of the static fields.

The static electric field breaks the symmetry so that we now observe both odd and even harmonics, and Fig. 5 shows

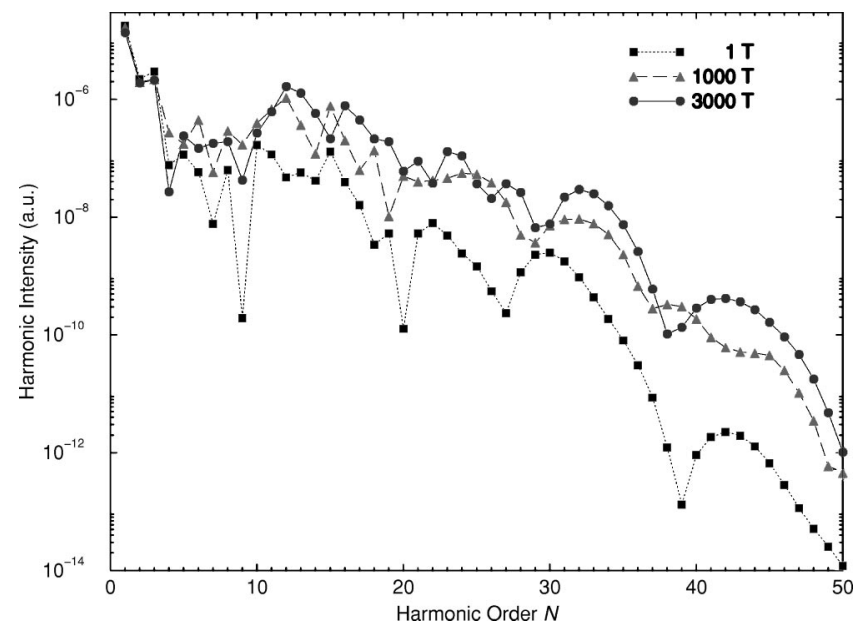

FIG. 5. The same as in Fig. 1, but in the presence of a parallel static electric field having strength $E_{S}=1 \mathrm{MV} / \mathrm{cm}$, for three values of the magnetic field induction: $B=1 \mathrm{~T}$ (squares), $1000 \mathrm{~T}$ (triangles), and $3000 \mathrm{~T}$ (circles). that the intensities of the even and odd harmonics are of the same order of magnitude. Figure 5 shows also that the harmonic spectrum is extended to higher harmonics: We now have two additional cutoffs, one at the 31 st harmonic, and the other at the 43rd harmonic. The positions of these cutoffs may be explained using a classical analysis, as we show in the next section. As in Fig. 1, the intensities of particular harmonics are increased by more than two orders of magnitude as the magnetic field is increased from 1 to $10^{3} \mathrm{~T}$. Thus by choosing appropriate particular values of both fields, it is possible to generate high-intensity, high-order harmonics. For example, the harmonics for $N=31,32$, and 33 shown in Fig. 5 all have higher efficiencies than harmonics $N>21$ beyond the cutoff harmonics in the absence of both fields (cf. Fig. 1).

Figure 6 shows the $B$-field dependence of the harmonic intensities for different groups of harmonics: (a) 12-15 [which are located in the plateau region in the absence of both static fields (cf. Fig. 1)], (b) 21-24 (which are located in the cutoff region for $B=0$ and $E_{S}=0$ ), (c) 30-33 (which are located in the region of the second cutoff, i.e., the first additional cutoff, which appears owing to the static electric field), and (d) 41-44 (which are located in the region of the third cutoff, i.e., the second additional cutoff, which appears owing to the static electric field). Figure 6 shows that the $B$ dependence of the harmonic intensity is more complex in the presence of the static electric field than in its absence (cf. Fig. 2). We still have maxima for particular values of $B$, but they are broader, and interference structures are present. (These interference structures occur even for $B=0$ in the presence of a strong static field [24-26].) Second, the highest maxima appear for $B<5000 \mathrm{~T}$. We see also in Fig. 6 that for a fixed value of the static electric field, maxima in the intensity of particular harmonics occur for a number of values of the static magnetic field. In the next section, using a classical analysis, we explain many of the features shown in Fig. 6.

\section{CLASSICAL ANALYSIS}

\section{A. Solutions of the classical equations of motion}

As is well known, the cutoff of the plateau in HHG in the absence of the static fields, i.e., $N_{\max } \hbar \omega=I_{p}+3.17 U_{p}$, can be obtained both classically $[17,18]$ and quantum mechanically $[15,20]$, based on the "three-step" model, which was described in the Introduction. According to the classical version of this model, the electron is born at time $t_{0}$ at the origin $\left[\mathbf{r}\left(t_{0}\right)=\mathbf{0}\right]$ with zero initial momentum. It then moves under the influence of the laser field, and, in our present case, the static fields. Solving Newton's equation for the electron, $m_{e} \ddot{\mathbf{r}}=-e\left[\mathbf{E}_{L}(t)+\mathbf{E}_{S}+\dot{\mathbf{r}} \times \mathbf{B}\right]$, one obtains the electron kinetic energy $E_{k}$ at the time $t_{1}$ when the electron returns to the origin. The maximum of this energy, and, therefore, the cutoff energy $N_{\max } \hbar \omega=I_{p}+E_{k, \max }$, is determined by the two conditions: $\mathbf{r}\left(t_{1}\right)=\mathbf{0}$ and $\partial E_{k} / \partial t_{0}=0$. The first condition implies that the electron, after the return time $\tau=t_{1}-t_{0}$, comes back to the atomic core, while the second condition optimizes the time $t_{0}$ at which the electron is "born" (i.e., excited to the continuum) to be that for which $E_{k}$ is maximal. In the case of a linearly polarized laser field and parallel static fields, Newton's equation separates, and, 

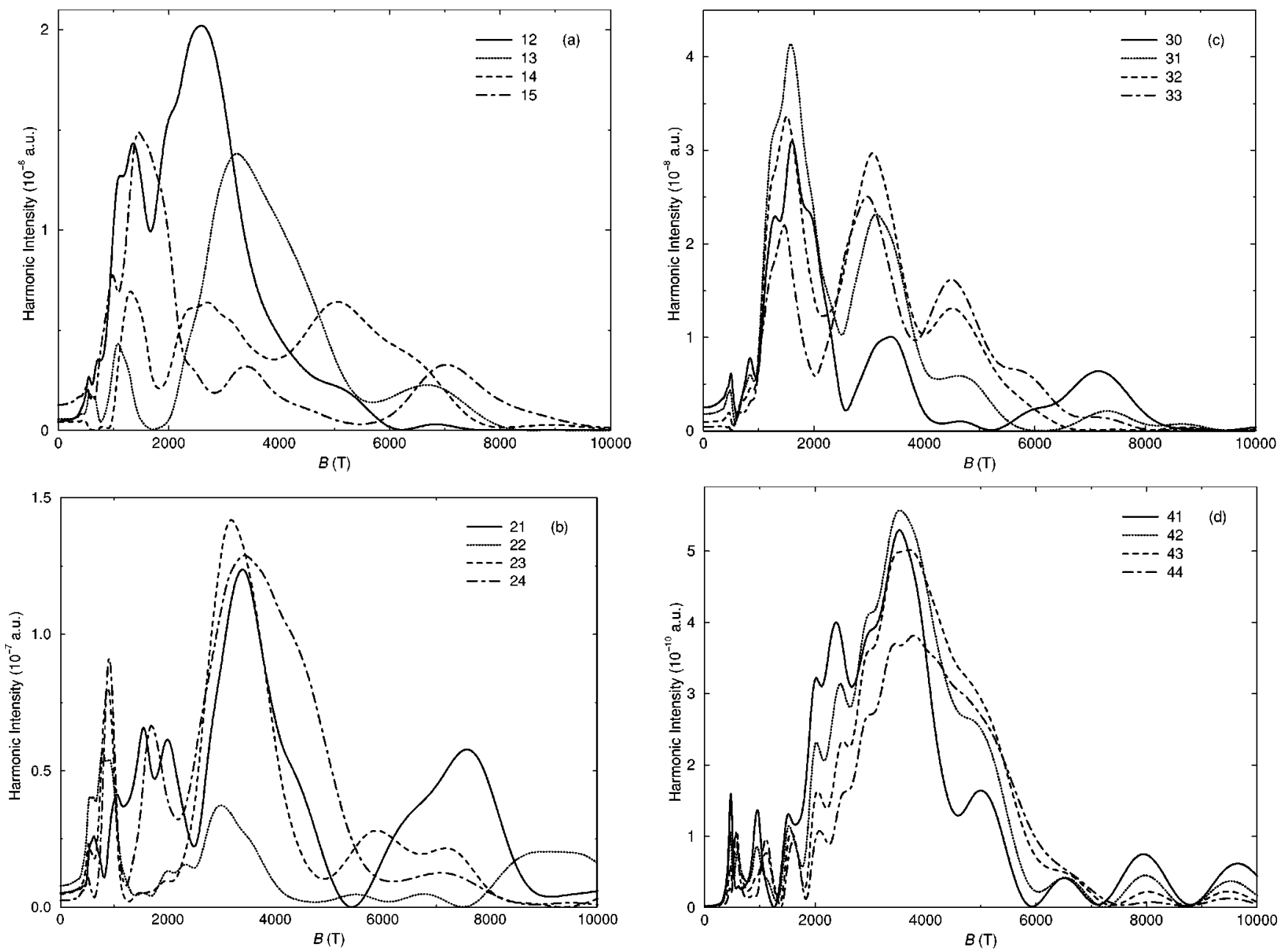

FIG. 6. Harmonic intensities as functions of the magnetic-field induction $B$ for fixed harmonic order: (a) $N=12-15$, (b) $21-24$, (c) $30-33$, and (d) 41-44. Results are for the $\mathrm{H}^{-}$ion in a $\mathrm{CO}_{2}$ laser with intensity $I=5 \times 10^{10} \mathrm{~W} / \mathrm{cm}^{2}$ and in a static electric field $E_{S}=1$ $\mathrm{MV} / \mathrm{cm}$.

for zero initial momentum, we have $m_{e} \dot{x}=e B y, m_{e} \dot{y}$ $=-e B x$, and $m_{e} \dot{z}=e\left[A_{L}(t)+A_{S}(t)-A_{L}\left(t_{0}\right)-A_{S}\left(t_{0}\right)\right]$, so that $E_{k}=m_{e} \dot{\mathbf{r}}^{2}(t) / 2=e^{2}\left\{\left[\mathbf{A}_{L}(t)+\mathbf{A}_{S}(t)-\mathbf{A}_{L}\left(t_{0}\right)-\mathbf{A}_{S}\left(t_{0}\right)\right]^{2}\right.$ $\left.+B^{2}\left(x^{2}+y^{2}\right)\right\} /\left(2 m_{e}\right)$. The electron rotates [46] in the plane perpendicular to the magnetic field with the cyclotron period $\tau_{B}=2 \pi / \omega_{B}$, while its parallel motion is determined by the laser field and the static electric field. The electron is back at the nucleus at time $t_{1}=t_{0}+\tau_{B}, t_{0}+2 \tau_{B}, \ldots$, if the condition $z\left(t_{1}\right)=0$ is fulfilled.

The solution of the equation $z\left(t_{1}\right)=0$, with the condition that the electron is born with zero initial momentum, i.e., $\dot{z}\left(t_{0}\right)=0$, leads to [see also Eqs. (A1), (A4), and (A6), and Refs. [25,43] for nonzero initial momentum]

$$
\int_{t_{0}}^{t_{1}} d t\left[A_{L}(t)+A_{S}(t)\right]=\left[A_{L}\left(t_{0}\right)+A_{S}\left(t_{0}\right)\right]\left(t_{1}-t_{0}\right) .
$$

For a laser field $\mathbf{E}_{L}(t)=E_{L} \sin \omega t \hat{\mathbf{z}}$, introducing the notations

$$
\varphi=\omega t_{1}, \quad \sigma=\frac{\omega \tau}{2}, \quad a(\sigma)=\sin \sigma
$$

$$
b(\sigma)=\cos \sigma-\frac{\sin \sigma}{\sigma}, \quad c(\sigma)=-\frac{E_{S}}{E_{L}} \sigma
$$

we can rewrite Eq. (8) as [43]

$$
a(\sigma) \sin (\varphi-\sigma)+b(\sigma) \cos (\varphi-\sigma)=c(\sigma) \text {. }
$$

In these variables, the electron's kinetic energy is given by

$$
E_{k}=8 U_{p}[a(\sigma) \sin (\varphi-\sigma)-c(\sigma)]^{2} .
$$

Equation (10) can be further rewritten as a quadratic equation in the variable $X \equiv \sin (\varphi-\sigma)$, and, therefore, for each $\tau$ one has two solutions for $X$ :

$$
X(\sigma)=\frac{1}{a^{2}+b^{2}}\left[a c \pm b\left(a^{2}+b^{2}-c^{2}\right)^{1 / 2}\right] .
$$

Introducing these solutions into Eq. (11), we obtain the harmonic $\operatorname{order} N$ as a function of the return time, i.e., $N(\omega \tau)$ $=\left[I_{p}+E_{k}(\tau)\right] / \hbar \omega$. The two solutions in Eq. (12) correspond to positive and negative values of $E_{L}(t)$ at the moment of ionization. For one solution, $\mathbf{E}_{L}\left(t_{0}\right)$ is parallel to the static electric field $\mathbf{E}_{S}$; for the other, it is antiparallel. In the ab- 


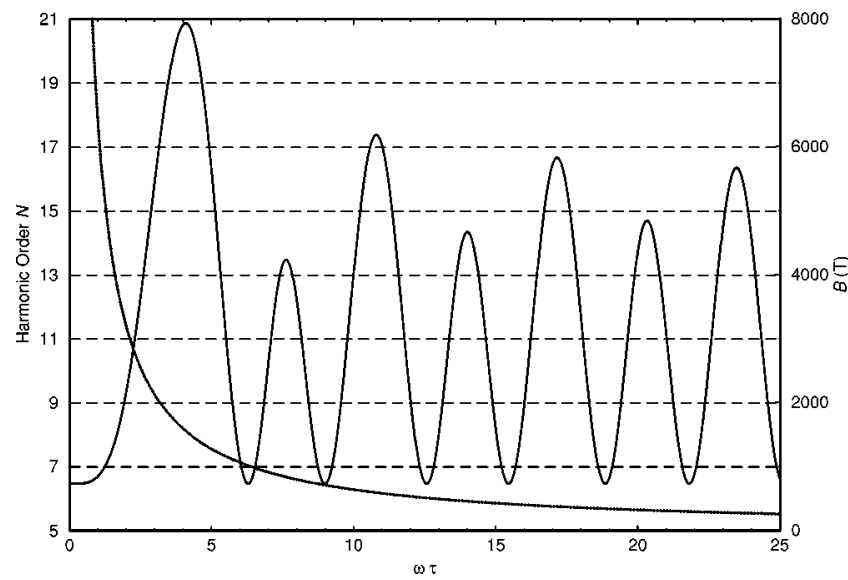

FIG. 7. Harmonic order $N$ as an oscillatory function of the dimensionless variable $\omega \tau$, where $\tau$ is the classical electron return time to the origin under the influence of the laser field [see Eqs. (9) and (13)]. The laser field and the $\mathrm{H}^{-}$ion parameters are as in Fig. 1. The monotonically decreasing curve, measured by the righthand-side ordinate, represents the magnetic induction $B$ as a function of $\omega \tau$ for $\tau=\tau_{B}$, where $\tau_{B}=2 \pi / \omega_{B}=2 \pi m_{e} /(e B)$ is the classical period for motion perpendicular to the magnetic field.

sence of the static electric field these two solutions coincide. Since for this latter case $c(\sigma)=0$, Eqs. (11) and (12) show that there is only one solution for $E_{k}$, which gives

$$
N \hbar \omega=I_{p}+\frac{8 a^{2} b^{2}}{a^{2}+b^{2}} U_{p} .
$$

The maximum value of the coefficient of $U_{p}$ in Eq. (13) is 3.17 , which corresponds to $N_{\max }$, the well-known cutoff of the HHG plateau $[15,17,18]$.

\section{B. Classical interpretation of quantum-mechanical HHG intensity revivals in a static $B$ field}

Useful information about the HHG process can be obtained by presenting $E_{k}$ as a function of the return time $\tau$ $=t_{1}-t_{0}$ [15]. In Fig. 7 we present the harmonic order $N$ $=\left(E_{k}+I_{p}\right) /(\hbar \omega)$ as a function of $\omega \tau$ for the $\mathrm{H}^{-}$ion, a $\mathrm{CO}_{2}$ laser with intensity $I=5 \times 10^{10} \mathrm{~W} / \mathrm{cm}^{2}$, and no static electric field (the same parameters as for Figs. 1 and 2). The oscillatory function $N=N(\omega \tau)$ [see Eqs. (13) and (9)] has a maximum at $N_{\max } \approx 21$ in the first optical cycle $(\omega \tau \leqslant 2 \pi)$, followed by infinitely many lower maxima [we present $N(\omega \tau)$ up to $\omega \tau=25]$. The intersections of the dashed horizontal lines $N=7,9, \ldots, 19$, with this oscillatory curve give the values of the return time $\tau$ of the ionized electron, which returns to the nucleus with the energies $7 \hbar \omega-I_{p}, 9 \hbar \omega$ $-I_{p}, \ldots, 19 \hbar \omega-I_{p}$, and which, therefore, can recombine with the atom emitting the 7th, 9th, ..., 19th harmonic, respectively. In the same figure (using the right-hand-side ordinate $B$ and the dotted curve), we present the magnetic induction $B$ as a function of $\omega \tau$ for $\tau=\tau_{B}$, where $\tau_{B}$ $=2 \pi / \omega_{B}=2 \pi m_{e} /(e B)$ is the classical period for motion perpendicular to the magnetic field. From such a presentation one can find the value of the magnetic field which corresponds to the process in which the ionized electron is back at the nucleus both in the parallel and in the perpendicular di-

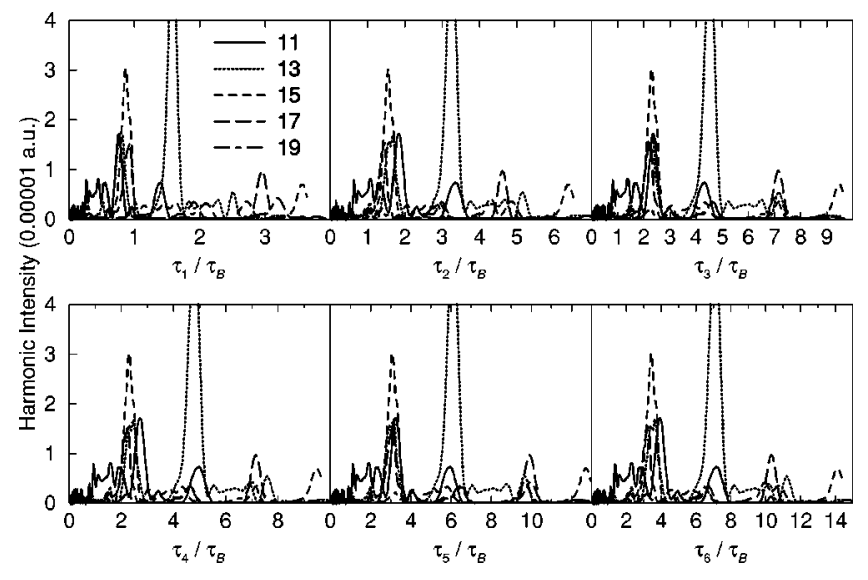

FIG. 8. Harmonic intensities for $11 \leqslant N \leqslant 19$ plotted in dimensionless units $\tau_{i}(N) / \tau_{B}, i=1, \ldots, 6$, where $\tau_{i}$ is the classically calculated period for the $i$ th return of the electron to the origin under the influence of the laser field, and $\tau_{B}$ is the cyclotron period. The laser field and the $\mathrm{H}^{-}$ion parameters are as in Fig. 1.

rection at the same time, having an energy sufficient to emit the $N$ th harmonic. As we will show below, these values of $B$ correspond to the maximum values of the harmonic intensities. For example, for the 15 th harmonic the shortest return time is for $\omega \tau_{1}=2.8718$, which corresponds to $B \approx 2200 \mathrm{~T}$. The next (second) return time $\tau_{2}$ for the 15 th harmonic is for $\omega \tau_{2}=5.1276$, which corresponds to $B \approx 1230 \mathrm{~T}$. Longer and longer return times correspond to one cyclotron period $\tau_{B}$ for smaller and smaller values of the magnetic induction $B$.

Let us now relate our quantum-mechanical results, presented in Figs. 1 and 2, to our classical orbit calculations, i.e., to the results presented in Fig. 7. For each harmonic $N$, $11 \leqslant N \leqslant 19$, we first calculated the corresponding classical return times $\tau_{i}(N), i=1, \ldots, 6$, where $i$ is defined below. For $N>13$ there are no intersections of the horizontal lines $N=15,17$, and 19 , with the curve $N(\omega \tau)$ in the first part of the second optical cycle $(2 \pi<\omega \tau<3 \pi)$. In this case, we have chosen both for $\tau_{3}$ and $\tau_{4}$ the value which corresponds to the second maximum (we do not have classical orbit solutions which return to the nucleus, but quantum mechanically the electronic wave packet amplitudes can still contribute to the process). Next, in the corresponding subplots in Fig. 8, we present our quantum-mechanical results for the harmonic intensity vs $B$ in terms of the $i$ th classical return time, $\tau_{i}(N) / \tau_{B} \equiv\left[e \tau_{i}(N) /\left(2 \pi m_{e}\right)\right] B, i=1, \ldots, 6$. Note that $\tau_{i}(N)$ does not depend on $B$ because the classical motion along the $z$ axis does not depend on it; thus $\tau_{i}(N) / \tau_{B}$ has a linear dependence on $B$, which stems from division by $\tau_{B}$. The dimensionless variable $\tau_{i}(N) / \tau_{B}$ measures the period $\tau_{i}(N)$ for the $i$ th return of the electron under the influence of the laser field in units of $\tau_{B}$. Whenever $\tau_{i}(N)$ is close to an integer multiple of $\tau_{B}$, there is a revival of intensity in the $N$ th harmonic; moreover the interval between revivals is approximately $\tau_{B}$. For example, all presented harmonics (except the 13th) have their largest intensity maximum close to $\tau_{1}=\tau_{B}$ (upper leftmost subplot in Fig. 8). The 11th harmonic maximum (solid curve) also has contributions from $\tau_{2}$ $\approx 2 \tau_{B}, \tau_{4} \approx 3 \tau_{B}$, and $\tau_{6} \approx 4 \tau_{B}$. For the 13th harmonic our calculations show contributions to the maximum from $\tau_{2}$ $\approx 3 \tau_{B}, \tau_{4} \approx 5 \tau_{B}, \tau_{5} \approx 6 \tau_{B}$, and $\tau_{6} \approx 7 \tau_{B}$, implying that the 


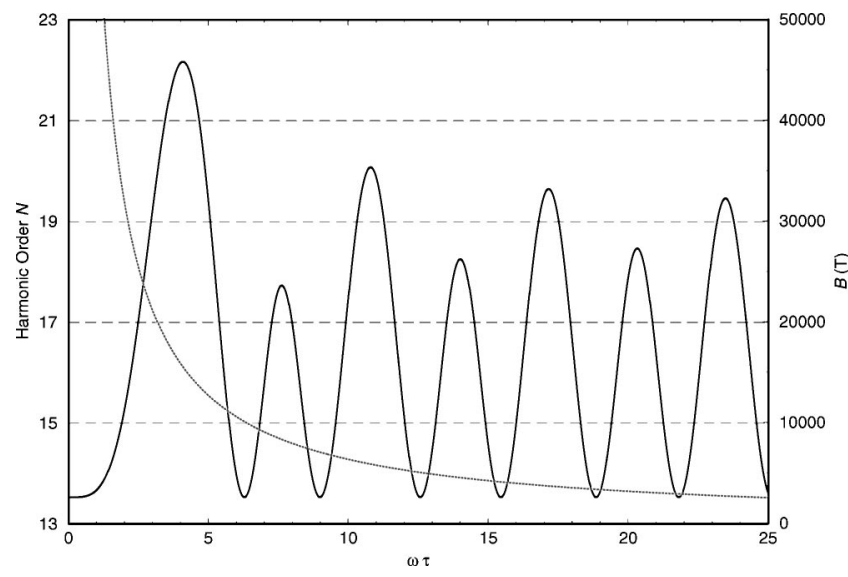

FIG. 9. The same as in Fig. 7, but for the laser field and the atomic parameters as in Fig. 3.

high intensity of the 13th harmonic may stem from constructive interference of contributions associated with a large number of classical orbits along the $z$-axis with return times $\tau$ that are multiples of $\tau_{B}$.

Similarly to Fig. 7, in Fig. 9 we present classical orbit calculations for the case of the Ar atom and a Nd:YAG laser with the same parameters as in Figs. 3 and 4. The maximum harmonic order is slightly larger than in Fig. 7 and intersections of the curve $N=N(\omega \tau)$ with the horizontal dashed lines appear for $15 \leqslant N \leqslant 21$. The corresponding magnetic fields $\left[B=\omega 2 \pi m_{e} /(e \omega \tau)\right.$ for $\left.\tau=\tau_{B}\right]$ are one order of magnitude larger than those presented in Fig. 7 in order that the cyclotron period for motion perpendicular to the $B$-field axis is comparable to the classical orbit period $\tau$. Figure 10 shows, similarly to Fig. 8, the results of our quantummechanical calculations for the harmonic intensity vs $B$ in terms of $\tau_{2}(N) / \tau_{B}$, for $15 \leqslant N \leqslant 21$. We see that the maxima for all harmonic intensities correspond to $\tau_{2} \approx \tau_{B}$, which means that, in this case, the main contribution to the harmonic revivals in the presence of the magnetic field occurs when the time for the second return of the ionized electron to the nucleus is equal to one cyclotron period. There are, of

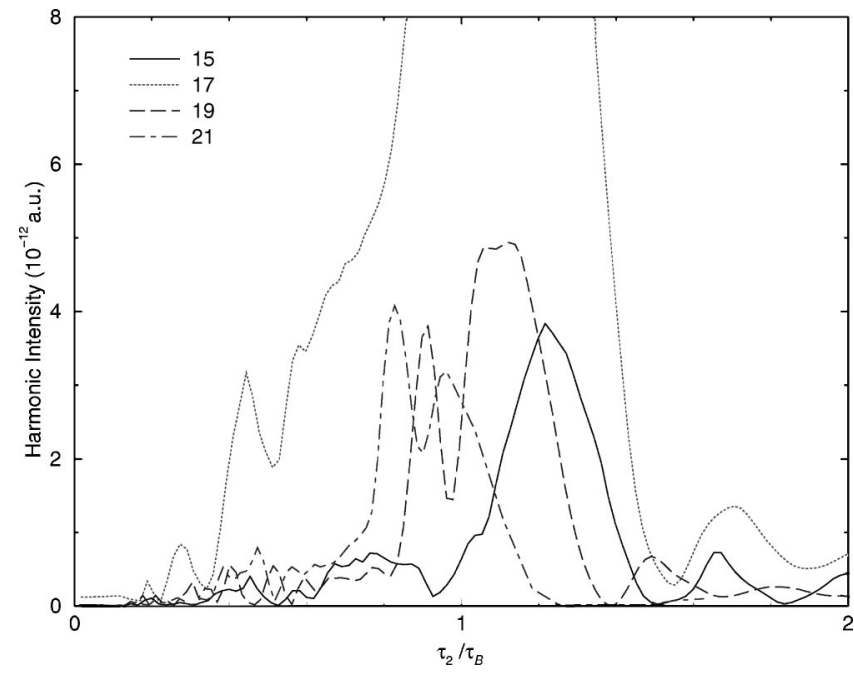

FIG. 10. The same as in Fig. 8, but for the laser field and the atomic parameters as in Fig. 3, for harmonic orders $15 \leqslant N \leqslant 21$, but for only one value of $i=2$.

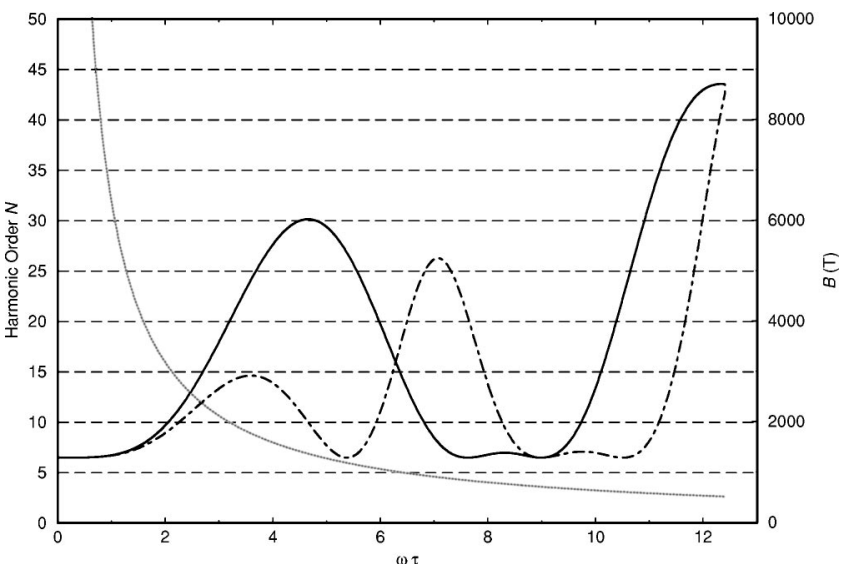

FIG. 11. The same as in Fig. 7, but in the presence of an additional parallel static electric field having strength $E_{S}=1 \mathrm{MV} / \mathrm{cm}$. The two solutions of Eqs. (9)-(12) are denoted by solid and dotdashed lines.

course, other $\tau_{i}$ for this case which are equal integer multiples of $\tau_{B}$, but we have presented in Fig. 10 what appears to us to be the most important classical orbit.

Generally, the intensity of harmonics peaks whenever the wave packet amplitudes for motion perpendicular to and along the $z$ axis coincide. The exact coincidence of the positions of harmonic intensity maxima, presented in Figs. 8 and 10 , with $\tau_{i}(N)=j \tau_{B}$, where $j$ is an integer, is not expected, for two reasons. First, quantum wave packets have a spatial width. Second, there are interferences between contributions associated with a large number of classical orbits along the $z$-axis with return times $\tau$ that are multiples of $\tau_{B}$.

\section{Classical interpretation of quantum-mechanical HHG features for parallel static $B$ and $E$ fields}

In the presence of a static electric field there are two solutions for the harmonic order $N$ as a function of the parameter $\omega \tau$ [see Eqs. (9)-(12)], which correspond to $\mathbf{E}_{L}\left(t_{0}\right)$ parallel or antiparallel to $\mathbf{E}_{S}$. The existence of these two classical solutions for $E_{S} \neq 0$ enables a more detailed test of the "three-step" model of HHG, which is one of the motivations for our consideration of the parallel static fields case. In Fig. 11 we present the two solutions for $N$ for the cases of parallel orientation (solid curve) and antiparallel orientation (dot-dashed curve). (See Figs. 7 and 9 which show only a single curve when $E_{S}=0$.) For a long enough return time ( $\omega \tau>12.41$ in the present case), the laser field cannot return the electron back to the nucleus because the influence of the static electric field, which is proportional to the return time, becomes too large. The maxima of the curves presented in Fig. 11 explain why the cutoffs in the harmonic spectrum which we obtained in our quantum-mechanical calculations (see Fig. 5) appear at $N=31$ and 43. In addition, as in Figs. 7 and 9 , the monotonically decreasing curve using the righthand-side ordinate presents the $B$ field corresponding to $\omega \tau$ $=\omega \tau_{B}$.

Our quantum-mechanical results, presented in Fig. 6, can be related to the classical calculations of Fig. 11 in a way similar to what we have presented for HHG in a $B$ field alone. We consider four particular harmonics: $N=12$, which lies on the plateau, and $N=25,34$, and 40, which are located at the first, second, and third cutoffs, respectively (cf. Fig. 6). 

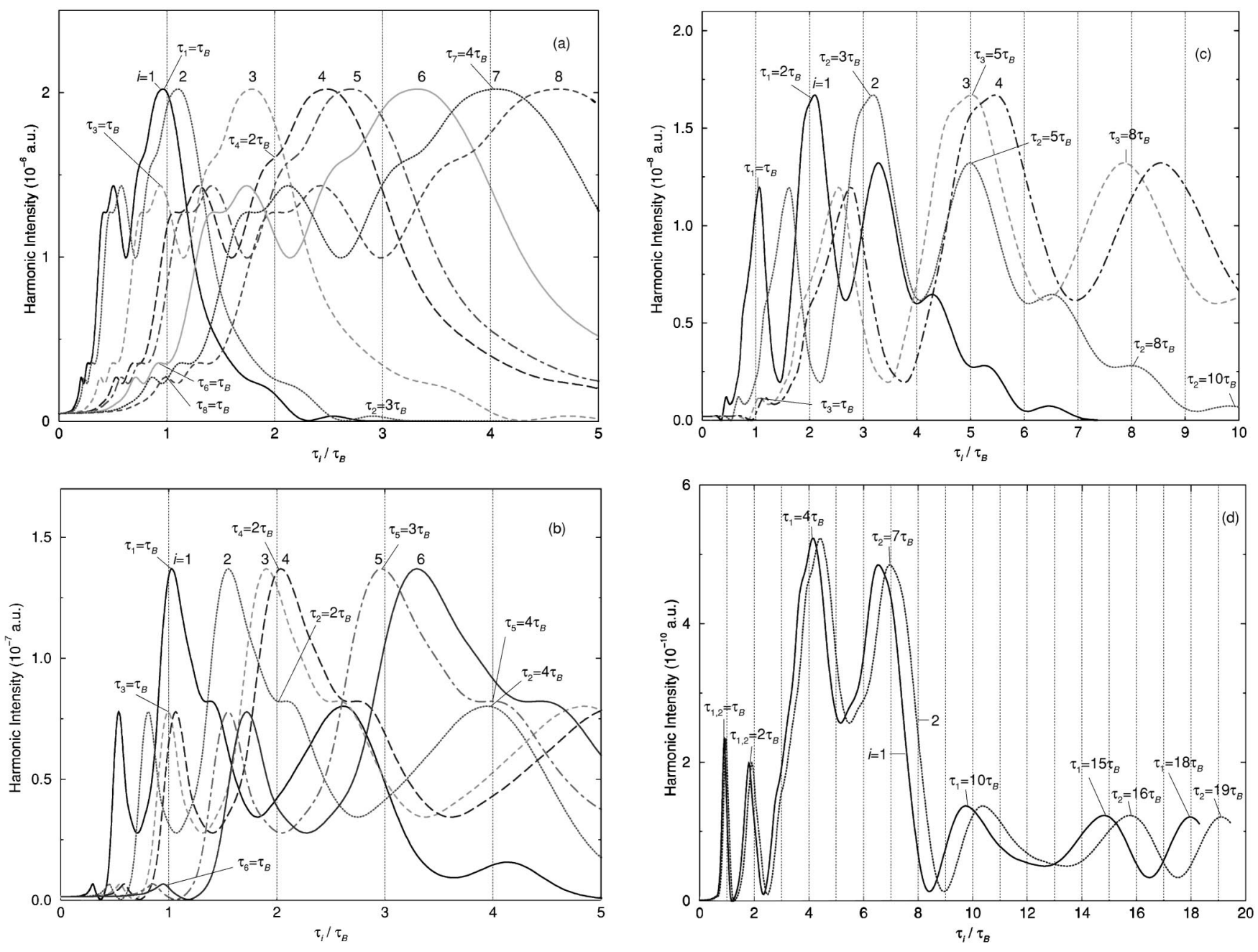

FIG. 12. The same as in Fig. 8, but in the presence of an additional parallel static electric field having strength $E_{S}=1 \mathrm{MV} / \mathrm{cm}$, and for the harmonic orders: (a) $N=12$, (b) 25, (c) 34, and (d) 40. For a given harmonic the quantum-mechanically calculated intensity is plotted vs the magnetic induction $B$ several times using the dimensionless variables $\tau_{i} / \tau_{B}$ for $i=1,2, \ldots$, where $\tau_{i}$ is the $i$ th classical return time. Harmonic intensity maxima occuring for $\tau_{i}(N)=j \tau_{B}$ are denoted in the figure, where $i$ and $j$ are integers, and $\tau_{B}$ is the cyclotron period. One sees from the figure how each feature in the $N$ th harmonic intensity curve may be associated with a particular classical orbit $i$.

For these harmonics we present the harmonic intensity as a function of the variable $\tau_{i}(N) / \tau_{B}$, where the return times $\tau_{i}(N)$ are obtained as those values of $\tau$ at which our results for the electron kinetic energy at the nucleus, $E_{k}(\tau)$, corresponds to the energy of the $N$ th harmonic, according to Eq. (11). This occurs when the $N$ th harmonic's horizontal line in Fig. 11 intersects the oscillating curves representing $\left[I_{p}\right.$ $\left.+E_{k}(\tau)\right] / \hbar \omega$. For $N=12$ we have 8 points of intersection, i.e., $\tau_{i}(12), i=1, \ldots, 8$, while for $N=25$ there are 6 such points. For $N=34$ we have only two intersections, at $\omega \tau_{3}$ $=11.14$ and $\omega \tau_{4}=12.11$, but there are also two maxima (at $\omega \tau_{1}=4.65$ and $\omega \tau_{2}=7.07$ ) which are lower than 34 , but which should also be taken into account because of the diffuse nature of electron wave packets as opposed to the precise nature of classical trajectories. Finally, for $N=40$, contributions of these maxima can be neglected, and only two return times contribute: $\omega \tau_{1}=11.57$ and $\omega \tau_{2}=12.3$. From Fig. 6(a) we see that, according to our quantum-mechanical calculations, the 12th harmonic has 8 maxima (although two of these are not fully developed: the curve exhibits only shoulder features). In Fig. 12(a) we present our quantummechanical results for the 12th harmonic's intensity as a function of the magnetic field induction $B$ but plotted 8 different ways, as functions of $\tau_{i} / \tau_{B}, i=1, \ldots, 8$. Our aim is to determine whether each of the 8 maximum in the quantum-mechanical intensity results may be associated with integer values of $\tau_{i} / \tau_{B}$ for one or more values of $i$. As expected, the main maximum is for $\tau_{1}=\tau_{B}$, i.e., for such values of the $B$ field for which the shortest return time of the electron under the driving influence of both the laser and static electric fields, is equal to one cyclotron period. The dashed $i=3$ curve shows that the second maximum [to the left of the highest one in Fig. 12(a)] comes from the third return time, i.e., from $\tau_{3}=\tau_{B}$. In Fig. 12(a) we have also denoted some other characteristic points. For example, the seventh return time also gives a contribution to the largest maximum (see curve 7, for which the maximum occurs at the point $\tau_{7}=4 \tau_{B}$ ), while the main contributions to the two lowest maxima at 550 and $720 \mathrm{~T}$ in Fig. 6(a) come from $\tau_{8}$ $=\tau_{B}$ and $\tau_{6}=\tau_{B}$, respectively. The results of a similar analysis for the 25th, 34th, and 40th harmonics are presented in Figs. 12(b), 12(c), and 12(d), respectively. We observe that some pronounced maxima appear to involve a constructive interference of electron wave packets with different re- 
turn times. For example, for $N=34$, in Fig. 12(c), the highest maximum has contributions from $\tau_{1}=2 \tau_{B}, \tau_{2}=3 \tau_{B}$, and $\tau_{3}=5 \tau_{B}$. This maximum is even higher than the maximum for the shortest return time $\tau_{1}=\tau_{B}$, perhaps because of these multiple contributions. For the cutoff harmonic of the third plateau [cf. $N=40$ in Fig. 12(d)], the major contributions come from two return times $\left(\omega \tau_{1}=11.57\right.$ and $\left.\omega \tau_{2}=12.3\right)$. We have thus demonstrated that maxima in the quantummechanically calculated harmonic intensities appear whenever one of the classically-calculated return times is equal to an integer multiple of the cyclotron period, i.e., $\tau_{i}=j \tau_{B}, i$ $=1,2, j=1,2, \ldots$. These results provide strong additional support for the validity of the "three-step" model of HHG.

\section{SUMMARY AND CONCLUSIONS}

In this paper we have presented a more detailed analysis of the possibility of controlling high-harmonic generation in a strong magnetic field than was presented in Ref. [40]. In order to confirm that our analysis is not specific to the $\mathrm{CO}_{2}$ laser frequency or the $\mathrm{H}^{-}$target considered in Ref. [40], we have considered here also the case of an Ar atom and a Nd:YAG laser field. Finally, in order to verify that our classical analysis based on the "three-step" model $[17,18]$ is valid even when the degeneracy of the classical orbits driven either parallel or antiparallel to the $z$ axis by the laser field is removed, we have considered also the case of HHG in parallel $E$ and $B$ fields. In our calculations the harmonic intensity is computed using the strong-field approximation combined with the saddle-point method. Also, the summation over intermediate Landau states is performed exactly.

We have shown that a strong magnetic field can increase the harmonic intensity considerably and that there are optimum values of the magnetic induction for which a particular harmonic is emitted with maximal efficiency. Using a $\mathrm{CO}_{2}$ laser and the $\mathrm{H}^{-}$ion, for example, we have shown that this maximum can be reached with experimentally available magnetic field strengths. We have also explained for all cases considered, using the classical "three-step" model, that the positions of these maxima correspond to such values of the magnetic induction for which an integer multiple of the classical period for motion perpendicular to the magnetic field is equal to the return time of the ionized electron wave packet to the nucleus under the driving influence of the laser and, if present, the static electric field. We interpret this fact to mean that the harmonic intensity has a maximum if the electronic wave packet is at the nucleus both in the parallel and in the perpendicular directions at the same time. While the static magnetic field (for the magnetic field inductions we are considering) does not affect the position of the HHG plateau cutoff significantly, the static electric field can introduce new plateaus with their own cutoffs, as has been shown theoretically for the case of $\mathrm{HHG}$ in only a static electric field $[25,26]$. Therefore, a properly chosen combination of the static electric and magnetic fields can increase both the harmonic intensity and the harmonic order. In the presence of the static electric field there are two solutions of the classical equations (one for parallel and the other for antiparallel orientations of the laser field and the static field at the moment of ionization), and the classical orbit analysis is more complicated. Nevertheless, using it we were able to explain both the cutoff positions and essentially each feature of the behavior of particular harmonics as a function of the magnetic field induction, $B$. Our results thus constitute strong additional support for the validity of the "three-step" model for HHG.

\section{ACKNOWLEDGMENTS}

This work has been supported in part by the National Science Foundation under Grant No. PHY-9722110.

\section{APPENDIX A}

In this appendix we consider the solution of the Schrödinger equation for an electron (with mass $m_{e}$ and charge $-e$, where $e>0$ ) in the simultaneous presence of a laser field $\mathbf{E}_{L}(t)$, a static electric field $\mathbf{E}_{S}$, and a static magnetic field $\mathbf{B}$. (SI units are used.) The total vector potential $\mathbf{A}(t)$ is given by

$$
\begin{gathered}
\mathbf{A}(t)=\mathbf{A}_{L}(t)+\mathbf{A}_{S}(t)+\mathbf{A}_{B}, \\
\mathbf{A}_{L}(t)=-\int^{t} d t^{\prime} \mathbf{E}_{L}\left(t^{\prime}\right), \\
\mathbf{A}_{S}(t)=-\mathbf{E}_{S} t, \quad \mathbf{A}_{B}=\frac{1}{2} \mathbf{B} \times \mathbf{r} .
\end{gathered}
$$

The Hamiltonian of this system in the radiation gauge is $H(t)=[\mathbf{p}+e \mathbf{A}(t)]^{2} /\left(2 m_{e}\right)$. The high-harmonic generation problem is formulated so that the interaction with the laser field is in the length gauge. Therefore, we make the following unitary transformation:

$$
\begin{aligned}
e^{i e \mathbf{a} \cdot \mathbf{r} / \hbar} H(t) e^{-i e \mathbf{a} \cdot \mathbf{r} / \hbar} & =\frac{1}{2 m_{e}}\{\mathbf{p}+e[\mathbf{A}(t)-\mathbf{a}]\}^{2}=H_{L}(t), \\
\mathbf{a} & =\mathbf{A}_{L}(t)+\mathbf{A}_{S}(t) .
\end{aligned}
$$

The obtained gauge is a mixture of the length gauge and the radiation gauge (for the magnetic field). The Schrödinger equation in this gauge has the form

$$
\begin{gathered}
{\left[i \hbar \frac{\partial}{\partial t}-H_{L}(t)\right]|\Psi(t)\rangle=0,} \\
H_{L}(t)=e \mathbf{r} \cdot\left(\mathbf{E}_{L}+\mathbf{E}_{S}\right)+\frac{1}{2 m_{e}}\left(\mathbf{p}+\frac{e}{2} \mathbf{B} \times \mathbf{r}\right)^{2} .
\end{gathered}
$$

The Hamiltonian $H_{L}(t)$ can be written as $H_{L}(t)=\mathbf{p}^{2} /\left(2 m_{e}\right)$ $+H_{E D}+H_{M D}+H_{N L}$, where $H_{E D}=e \mathbf{r} \cdot\left(\mathbf{E}_{L}+\mathbf{E}_{S}\right)$ is the electric-dipole interaction, $H_{M D}=e \mathbf{B} \cdot \mathbf{L} /\left(2 m_{e}\right)$, with $\mathbf{L}=\mathbf{r} \times \mathbf{p}, \quad$ is the magnetic-dipole interaction, and $H_{N L}=e^{2}(\mathbf{B} \times \mathbf{r})^{2} /\left(8 m_{e}\right)$ is the nonlinear diamagnetic term [47]. The solution of the above Schrödinger equation, in the special case of parallel fields

$$
\mathbf{E}_{L}(t)=E_{L}(t) \hat{\mathbf{z}}, \quad \mathbf{E}_{S}=E_{S} \hat{\mathbf{z}}, \quad \mathbf{B}=B \hat{\mathbf{z}}, \quad \mathbf{A}_{B}=\frac{B}{2}(-y, x, 0),
$$

can be written, in cylindrical coordinates $(\rho, \phi, z)$ in the form 


$$
\begin{aligned}
\Psi_{q n m}(\rho, \phi, z, t)= & (2 \pi)^{-1 / 2} \exp \left[i\left(q+\frac{e}{\hbar}\left[A_{L}(t)+A_{S}(t)\right]\right) z\right] \\
& \times \exp \left\{-i\left[q \alpha(t)+U(t) / \hbar+\hbar q^{2} t /\left(2 m_{e}\right)\right]\right\} \\
& \times \Phi_{n m}(\rho, \phi) \exp \left(-i E_{n m} t / \hbar\right),
\end{aligned}
$$

where $q$ is the $z$ component of the electron momentum,

$$
\begin{gathered}
\alpha(t)=\frac{e}{m_{e}} \int^{t} d t^{\prime}\left[A_{L}\left(t^{\prime}\right)+A_{S}\left(t^{\prime}\right)\right], \\
U(t)=\frac{e}{2 m_{e}} \int^{t} d t^{\prime}\left[A_{L}\left(t^{\prime}\right)+A_{S}\left(t^{\prime}\right)\right]^{2},
\end{gathered}
$$

and $\Phi_{n m}(\rho, \phi)$ satisfies the stationary Schrödinger equation

$$
\begin{gathered}
\left\{-\frac{\hbar^{2}}{2 m_{e}}\left[\frac{1}{\rho} \frac{\partial}{\partial \rho}\left(\rho \frac{\partial}{\partial \rho}\right)+\frac{1}{\rho^{2}} \frac{\partial^{2}}{\partial \phi^{2}}\right]+\frac{e B}{2 m_{e}} L_{z}\right. \\
\left.+\frac{e^{2} B^{2}}{8 m_{e}} \rho^{2}\right\} \Phi_{n m}(\rho, \phi)=E_{n m} \Phi_{n m}(\rho, \phi) .
\end{gathered}
$$

The wave functions $\Phi_{n m}(\rho, \phi)$ are solutions of the Schrödinger equation for an electron in the magnetic field only (i.e., the so-called Landau states [42])

$$
\begin{aligned}
& \Phi_{n m}(\rho, \phi)= C_{n m} \exp (i m \phi) \gamma^{|m| / 2} \rho^{|m|} L_{n}^{|m|}\left(\gamma \rho^{2}\right) \\
& \times \exp \left(-\frac{1}{2} \gamma \rho^{2}\right), \\
& C_{n m}^{2}=\frac{\gamma n !}{\pi(n+|m|) !}, \\
& E_{n m}= {\left[n+\frac{1}{2}(|m|+m+1)\right] \hbar \omega_{B}, } \\
& \omega_{B}=\frac{e B}{m_{e}}, \quad \gamma=\frac{e B}{2 \hbar} .
\end{aligned}
$$

In Eq. (A8) the functions $L_{n}^{|m|}\left(\gamma \rho^{2}\right)$ are associated Laguerre polynomials $[48,49], n$ is the radial quantum number, $m$ is the azimuthal component of the angular momentum, $\omega_{B}$ is the cyclotron frequency, and the wave functions are orthonormalized according to $\left\langle\Psi_{n m q} \mid \Psi_{n^{\prime} m^{\prime} q^{\prime}}\right\rangle=\delta_{n n^{\prime}} \delta_{m m^{\prime}} \delta(q$ $\left.-q^{\prime}\right)$. The time-dependent Green's function which corresponds to the solution (A5), is

$$
\begin{aligned}
G_{L}\left(\mathbf{r}, t ; \mathbf{r}^{\prime}, t^{\prime}\right) \\
=-\frac{i}{\hbar} \theta\left(t-t^{\prime}\right) \int_{-\infty}^{\infty} d q \sum_{n=0}^{\infty} \sum_{m=-\infty}^{\infty} \Psi_{n m q}(\rho, \phi, z, t) \\
\quad \times \Psi_{n m q}^{*}\left(\rho^{\prime}, \phi^{\prime}, z^{\prime}, t^{\prime}\right) .
\end{aligned}
$$

\section{APPENDIX B}

The time-dependent dipole matrix element, introduced in Sec. II, contains the following matrix elements between the wavefunction given in Appendix $\mathrm{A}$ and the atomic ground state $\left|u_{0}\right\rangle$,

$$
\begin{aligned}
d_{n m q} & =\left\langle u_{0}|z| n m q\right\rangle \\
& =\int_{-\infty}^{\infty} d z \int_{0}^{\infty} d \rho \rho \int_{0}^{2 \pi} d \phi u_{0}^{*}(\rho, \phi, z) z \Psi_{n m q}(\rho, \phi, z, 0) .
\end{aligned}
$$

We will consider ground states which do not depend on the polar angle $\phi$. In this case, the integration over $\phi$ gives: $\int_{0}^{2 \pi} d \phi \exp (i m \phi)=2 \pi \delta_{m, 0}$, so that, according to Eqs. (A5) and (A8), $d_{n m q}=\delta_{m, 0} f_{n}(q)$, where

$$
\begin{aligned}
f_{n}(q)= & (2 \gamma)^{1 / 2} \int_{-\infty}^{\infty} d z \int_{0}^{\infty} d \rho \rho u_{0}^{*}(\rho, z) z \\
& \times \exp (i q z) L_{n}\left(\gamma \rho^{2}\right) \exp \left(-\frac{1}{2} \gamma \rho^{2}\right) .
\end{aligned}
$$

For spherically symmetric ground states, $u_{0}(\rho,-z)$ $=u_{0}(\rho, z)=u_{0}\left(\sqrt{\rho^{2}+z^{2}}\right)$, so that

$$
\begin{aligned}
f_{n}(q)= & (2 \gamma)^{1 / 2} 2 i \int_{0}^{\infty} d z z \int_{0}^{\infty} d \rho \rho u_{0}^{*}\left(\sqrt{\rho^{2}+z^{2}}\right) L_{n}\left(\gamma \rho^{2}\right) \\
& \times \sin q z \exp \left(-\frac{1}{2} \gamma \rho^{2}\right) .
\end{aligned}
$$

We consider ground states of the form

$$
u_{0}(r)=\left(a+\frac{b}{r}\right) \exp (-c r)=\left(b-a \frac{\partial}{\partial c}\right) \frac{\exp (-c r)}{r}
$$

For $b=0$ this state corresponds to the hydrogen atom ground state, while for $a=0$ it describes the ground state of the $\mathrm{H}^{-}$ ion. Using the formula (3.961) from Ref. [48], the integral over $z$ in Eq. (B3) can be carried out explicitly, with the result

$$
\int_{0}^{\infty} d z z \frac{\exp \left(-c \sqrt{\rho^{2}+z^{2}}\right)}{\sqrt{\rho^{2}+z^{2}}} \sin q z=\frac{q \rho}{\sqrt{q^{2}+c^{2}}} K_{1}\left(\rho \sqrt{q^{2}+c^{2}}\right) .
$$

Using the relation [48]: $(\partial / \partial c) K_{0}\left(\rho \sqrt{q^{2}+c^{2}}\right)$ $=-\rho c K_{1}\left(\rho \sqrt{q^{2}+c^{2}}\right) / \sqrt{q^{2}+c^{2}}$, we obtain

$$
\begin{aligned}
f_{n}(q)= & (2 \gamma)^{1 / 2} 2 i q\left(b-a \frac{\partial}{\partial c}\right)\left(-\frac{1}{c} \frac{\partial}{\partial c}\right) \\
& \times \int_{0}^{\infty} d \rho \rho L_{n}\left(\gamma \rho^{2}\right) K_{0}\left(\rho \sqrt{q^{2}+c^{2}}\right) \\
& \times \exp \left(-\frac{1}{2} \gamma \rho^{2}\right) .
\end{aligned}
$$

The Laguerre polynomial $L_{n}$ can be expressed as a finite sum $[48,49]$

$$
L_{n}(x)=\sum_{k=0}^{n}(-1)^{k}\left(\begin{array}{c}
n \\
n-k
\end{array}\right) \frac{x^{k}}{k !},
$$

so that Eq. (B6) can be written as 


$$
\begin{aligned}
f_{n}(q)= & (2 \gamma)^{1 / 2} 2 i q\left(b-a \frac{\partial}{\partial c}\right)\left(-\frac{1}{c} \frac{\partial}{\partial c}\right) \\
& \times \sum_{k=0}^{n} \frac{(-1)^{k}}{k !}\left(\begin{array}{c}
n \\
n-k
\end{array}\right) \gamma^{k} F_{k}(c),
\end{aligned}
$$

where the integral $F_{k}(c)$ can be solved using formula (6.631.3) from Ref. [48],

$$
\begin{aligned}
F_{k}(c) & =\int_{0}^{\infty} d x x^{2 k+1} K_{0}(\beta x) \exp \left(-\frac{1}{2} \gamma x^{2}\right) \\
& =\frac{(k !)^{2}}{2 \beta}\left(\frac{2}{\gamma}\right)^{k+1 / 2} W_{-k-1 / 2,0}\left(\frac{\beta^{2}}{2 \gamma}\right) \exp \left(\frac{\beta^{2}}{4 \gamma}\right),
\end{aligned}
$$

where $\beta=\sqrt{q^{2}+c^{2}}$. Using the following connection between the Whittaker confluent hypergeometric function $W_{\mu, \nu}(z)$ and the Tricomi confluent hypergeometric function $\Psi(a, c ; z)[48,49]$,

$$
z^{-1 / 2} e^{z / 2} W_{-k-1 / 2,0}(z)=\Psi(k+1,1 ; z),
$$

we obtain

$$
\begin{aligned}
f_{n}(q)= & (2 \gamma)^{1 / 2} \frac{i q}{\gamma}\left(b-a \frac{\partial}{\partial c}\right)\left(-\frac{1}{c} \frac{\partial}{\partial c}\right) \\
& \times \sum_{k=0}^{n} \frac{(-2)^{k} n !}{(n-k) !} \Psi(k+1,1 ; z), \quad z=\frac{q^{2}+c^{2}}{2 \gamma} .
\end{aligned}
$$

The derivatives over $c$ in the above equation can be carried out explicitly using the following property of the $\Psi$ function [49]:

$$
\begin{gathered}
\frac{d^{n}}{d z^{n}} \Psi(a, b ; z)=(-1)^{n}(a)_{n} \Psi(a+n, b+n ; z), \\
(a)_{n}=a(a+1) \cdots(a+n-1)=\frac{\Gamma(a+n)}{\Gamma(a)} .
\end{gathered}
$$

The final result is therefore

$$
\begin{aligned}
f_{n}(q)= & (2 \gamma)^{1 / 2} \frac{i q}{\gamma^{2}} \sum_{k=0}^{n}\left(\begin{array}{l}
n \\
k
\end{array}\right)(-2)^{k}(k+1) ! b \Psi(k+2,2 ; z) \\
& \left.+\frac{2 a c}{q^{2}+c^{2}}(k+2) z \Psi(k+3,3 ; z)\right]
\end{aligned}
$$

where the parameter $z=\left(q^{2}+c^{2}\right) /(2 \gamma) \propto\left(B / B_{0}\right)^{-1}$, where $B_{0}=2.3505 \times 10^{5} \mathrm{~T}$. For the current experimentally available values of $B$, one has that $B \ll B_{0}$, so that $z \gg 1$. In this case we can simplify Eq. (B13) using the following asymptotic expansion of the $\Psi$ function [Eq. (13.5.2) in Ref. [49]]:

$$
\begin{aligned}
\Psi(k+s, s ; z)= & z^{-k-s}\left\{\sum_{r=0}^{R-1} \frac{(k+s)_{r}(k+1)_{r}}{r !}(-z)^{-r}\right. \\
& \left.+O\left(|z|^{-R}\right)\right\}, \quad s=2,3
\end{aligned}
$$

\section{APPENDIX C}

In order to obtain the time-dependent dipole matrix element, we have to compute the infinite sum

$$
G\left(q_{1}, q_{2}\right)=\sum_{n=0}^{\infty} f_{n}\left(q_{1}\right) f_{n}\left(q_{2}\right) \exp \left(-i n \omega_{B} \tau\right)
$$

where the functions $f_{n}$ are linear combinations [see Eq. (B13)] of the confluent hypergeometric function of the second type, or the Tricomi psi function $\Psi(a, b ; z)$. For small values of the magnetic induction $B$ one should take into account a large number of Landau states $n$, which causes problems in the numerical calculations. Therefore, we present here a different approach in which the summation over $n$ is performed analytically. Using the following integral representation of the function $\Psi(a, b ; z)$ [Eq. (13.2.5) in Ref. [49]]:

$$
\begin{aligned}
\Gamma(a) \Psi(a, b ; z)= & \int_{0}^{\infty} d t e^{-z t} t^{a-1}(1+t)^{b-a-1}, \\
& \operatorname{Re}(a)>0, \operatorname{Re}(z)>0,
\end{aligned}
$$

we obtain

$$
\begin{gathered}
b(k+1) ! \Psi(k+2,2 ; z)+\frac{2 a c}{q^{2}+c^{2}}(k+2) ! z \Psi(k+3,3 ; z) \\
=\int_{0}^{\infty} d t e^{-z t}\left(\frac{t}{1+t}\right)^{k+1}\left(b+\frac{2 a c}{q^{2}+c^{2}} z t\right) .
\end{gathered}
$$

Using the binomial expansion formula we get

$$
\sum_{k=0}^{n}\left(\begin{array}{l}
n \\
k
\end{array}\right)\left(\frac{-2 t}{1+t}\right)^{k}=\left(\frac{1-t}{1+t}\right)^{n}
$$

so that

$$
f_{n}(q)=(2 \gamma)^{1 / 2} \frac{i q}{\gamma^{2}} \int_{0}^{\infty} d t e^{-z t} \frac{t}{1-t}\left(\frac{1-t}{1+t}\right)^{n}\left(b+\frac{a c}{\gamma} t\right) .
$$

Introducing Eq. (C5) into Eq. (C1), and taking into account the formula $\sum_{n=0}^{\infty} z^{n}=1 /(1-z),|z|<1$, we obtain

$$
\begin{aligned}
G\left(q_{1}, q_{2}\right)= & \frac{2 q_{1} q_{2}}{\gamma^{3}} \int_{0}^{\infty} d x e^{-z_{1} x} \frac{x}{1+x}\left(b+\frac{a c}{\gamma} x\right) \\
& \times \int_{0}^{\infty} d y e^{-z_{2} y} \frac{y}{1+y} \frac{b+(a c / \gamma) y}{1-z}
\end{aligned}
$$




$$
z_{j}=\frac{q_{j}^{2}+c^{2}}{2 \gamma}, \quad z=\frac{1-x}{1+x} \frac{1-y}{1+y} \exp \left(-i \omega_{B} \tau\right) .
$$

The condition $|z|<1$ is fulfilled for $x \neq 0$ or $y \neq 0$. For $x$ $=y=0$ one must take into account that $\tau$ in Eq. (C1) occurs as a part of the Green's function (A9) and should be replaced by $\tau-i \varepsilon, \varepsilon \rightarrow 0^{+}$, so that $\left|\exp \left(-i \omega_{B} \tau\right)\right|=\exp \left(-\varepsilon \omega_{B}\right)<1$. After the substitution $z_{1} x \rightarrow y, z_{2} y \rightarrow x$, and using the notation

$$
\beta_{j}=\frac{2 a c}{p_{j}}, \quad p_{j}=q_{j}^{2}+c^{2}, \quad \beta=\frac{1-\exp \left(-i \omega_{B} \tau\right)}{2 \gamma},
$$

we obtain from Eq. (C6)

$$
\begin{aligned}
G\left(q_{1}, q_{2}\right)= & \frac{8 q_{1} q_{2}}{p_{1} p_{2}} \int_{0}^{\infty} d y \frac{y e^{-y}\left(b+\beta_{1} y\right)}{p_{1}-\gamma \beta\left(p_{1}-2 \gamma y\right)} \\
& \times \int_{0}^{\infty} d x \frac{x e^{-x}\left(b+\beta_{2} x\right)}{x+z}
\end{aligned}
$$

where now

$$
z=\frac{p_{2}}{2} \frac{2 y+\beta\left(p_{1}-2 \gamma y\right)}{p_{1}-\gamma \beta\left(p_{1}-2 \gamma y\right)} .
$$

Because of the presence of the factors $y \exp (-y)$ and $x \exp (-x)$, it is convinient to compute the integrals in Eq. (C8) using the generalized Gauss-Laguerre quadrature. Furthermore, using one of the definitions of the exponential integral of a complex argument $z$ and integer order $n[49,50]$

$$
E_{n}(z)=\frac{e^{-z}}{\Gamma(n)} \int_{0}^{\infty} d t \frac{e^{-t} t^{n-1}}{z+t}, \quad|\arg z|<\pi, \quad n=1,2, \ldots,
$$

the integral over $x$ in Eq. (C8) gives

$$
\int_{0}^{\infty} d x \frac{x e^{-x}\left(b+\beta_{2} x\right)}{x+z}=b e^{z} E_{2}(z)+2 \beta_{2} e^{z} E_{3}(z) .
$$

The exponential integral $E_{n}(z)$ can be efficiently computed using the subroutine ZEXINT from Ref. [50], while the remaining integral over $y$ can be computed using a generalized Gauss-Laguerre quadrature with the weight function $y e^{-y}$.
[1] N. B. Delone and V. P. Krainov, Multiphoton Processes in Atoms (Springer, Berlin, 1994).

[2] F. H. M. Faisal, Theory of Multiphoton Processes (Plenum, New York, 1987).

[3] M. H. Mittleman, Introduction to the Theory of Laser-Atom Interactions, 2nd ed. (Plenum, New York, 1993).

[4] Atoms in Intense Laser Fields, edited by M. Gavrila (Academic, Boston, 1992).

[5] Super-Intense Laser-Atom Physics, Vol. 316 of NATO Advanced Science Institutes Series, Series B: Physics, edited by B. Piraux, A. L'Huillier, and K. Rzążewski (Plenum, New York, 1993).

[6] Super-Intense Laser-Atom Physics IV, Proceedings of the NATO Advanced Research Workshop on SILAP IV, Moscow, Russia, 1995, edited by H. G. Muller and M. V. Fedorov (Kluwer, Dordrecht, 1996).

[7] Multiphoton Processes 1996, Proceedings of the 7th International Conference on Multiphoton Processes held in GarmischPartenkirchen, edited by P. Lambropoulos and H. Walther, Institute of Physics Conference Series No. 154 (IOP, Bristol, 1997).

[8] L. F. DiMauro and P. Agostini, Adv. At., Mol., Opt. Phys. 35, 79 (1995)

[9] W. Becker, A. Lohr, and M. Kleber, Quantum Semiclassic. Opt. 7, 423 (1995).

[10] M. Protopapas, C. H. Keitel, and P. L. Knight, Rep. Prog. Phys. 60, 389 (1997).

[11] C. J. Joachain, M. Dörr, and N. J. Kylstra, Comments At. Mol. Phys. 33, 247 (1997).

[12] F. Ehlotzky, A. Jaroń, and J. Z. Kamiński, Phys. Rep. 297, 63 (1998).

[13] P. Lambropoulos, P. Maragakis, and J. Zhang, Phys. Rep. 305, 203 (1998).

[14] A. L'Huillier, K. J. Schafer, and K. C. Kulander, J. Phys. B 24, 3315 (1991).
[15] M. Lewenstein, Ph. Balcou, M. Yu. Ivanov, A. L'Huillier, and P. B. Corkum, Phys. Rev. A 49, 2117 (1994); A. L'Huillier, M. Lewenstein, P. Salières, Ph. Balcou, M. Yu. Ivanov, J. Larsson, and C. G. Wahlstrom, ibid. 48, R3433 (1993).

[16] P. Salières, A. L'Huillier, Ph. Antoine, and M. Lewenstein, Adv. At., Mol., Opt. Phys. 41, 83 (1999).

[17] P. B. Corkum, Phys. Rev. Lett. 71, 1994 (1993).

[18] K. C. Kulander, K. J. Schafer, and J. L. Krause, in SuperIntense Laser-Atom Physics (Ref. [5]), p. 95.

[19] S. Long, W. Becker, and J. K. McIver, Phys. Rev. A 52, 2262 (1995).

[20] D. B. Milošević and B. Piraux, Phys. Rev. A 54, 1522 (1996); M. B. Gaarde, A. L'Huillier, and M. Lewenstein, ibid. 54, 4236 (1996).

[21] A. de Bohan, Ph. Antoine, D. B. Milošević, and B. Piraux, Phys. Rev. Lett. 81, 1837 (1998).

[22] M. Ivanov, P. B. Corkum, T. Zuo, and A. Bandrauk, Phys. Rev. Lett. 74, 2933 (1995); Ph. Antoine, B. Piraux, D. B. Milošević, and M. Gajda, Phys. Rev. A 54, R1761 (1996); Ph. Antoine, D. B. Milošević, M. B. Gaarde, P. Salières, A. L'Huillier, and M. Lewenstein, ibid. 56, 4960 (1997).

[23] J. B. Watson, A. Sanpera, X. Chen, and K. Burnett, Phys. Rev. A 53, R1962 (1996); A. Sanpera, J. B. Watson, M. Lewenstein, and K. Burnett, ibid. 54, 4320 (1996); S. De Luca and E. Fiordilino, J. Phys. B 29, 3227 (1996); E. C. Jarque and L. Plaja, ibid. 31, 1687 (1998).

[24] M. Q. Bao and A. F. Starace, Phys. Rev. A 53, R3723 (1996).

[25] A. Lohr, W. Becker, and M. Kleber, Laser Phys. 7, 615 (1997).

[26] B. Wang, X. Li, and P. Fu, J. Phys. B 31, 1961 (1998); Chin. J. Phys. 15, 195 (1998).

[27] T. Zuo, A. Bandrauk, M. Ivanov, and P. B. Corkum, Phys. Rev. A 51, 3991 (1995).

[28] T. Zuo and A. D. Bandrauk, J. Nonlinear Opt. Phys. Mater. 4, 533 (1995) 
[29] A. D. Bandrauk, J. Ruel, T. Zuo, and H. Yu, Int. J. Quantum Chem. 64, 613 (1997).

[30] J.-P. Connerade and C. H. Keitel, Phys. Rev. A 53, 2748 (1996).

[31] Y. I. Salamin and F. H. M. Faisal, Phys. Rev. A 58, 3221 (1998).

[32] R. H. Garstang, Rep. Prog. Phys. 40, 105 (1977).

[33] C. W. Clark, K. T. Lu, and A. F. Starace, in Progress in Atomic Spectroscopy, Part C, edited by H. J. Beyer and H. Kleinpoppen (Plenum, New York, 1984), p. 247. See especially Chap. 7, Secs. 2 and 6.

[34] M. R. C. McDowell and M. Zarcone, Adv. At. Mol. Phys. 21, 255 (1985).

[35] H. Friedrich and D. Wintgen, Phys. Rep. 183, 37 (1989).

[36] Atoms in Strong Fields, Proceedings of a NATO Advanced Study Institute on Atoms in Strong Fields, 1988, Island of Kos, Greece, edited by C. A. Nicolaides, C. W. Clark, and M. H. Nayfeh, Vol. 212 of NATO Advanced Science Institutes Series, Series B: Physics (Plenum, New York, 1990).

[37] Yu. B. Kudasov, A. I. Bykov, M. I. Dolotenko, N. P. Kolokol'chikov, M. P. Monakhov, I. M. Markevtsev, V. V. Platonov, V. D. Selemir, O. M. Tatsenko, A. V. Filippov, A. G. Volkov, A. A. Povzner, P. V. Bayankin, and V. G. Guk, Pis'ma Zh. Eksp. Teor. Fiz. 68, 326 (1998) [JETP Lett. 68, 350 (1998)].

[38] A. Pukhov and J. Meyer-ter-Vehn, Phys. Rev. Lett. 76, 3975 (1996); M. Borghesi, A. J. MacKinnon, R. Gaillard, O. Willi, A. Pukhov, and J. Meyer-ter-Vehn, ibid. 80, 5137 (1998); M. Borghesi, A. J. MacKinnon, R. J. Taylor, and O. Willi, ibid. 81, 4274 (1998); J. Fuchs, G. Malka, J. C. Adam, F. Amiranoff, S. D. Baton, N. Blanchot, A. Héron, G. Laval, J. L. Miquel, P. Mora, H. Pépin, and C. Rousseaux, ibid. 80, 1658 (1998); 81, 4275 (1998).

[39] Y. Horovitz, S. Eliezer, Z. Henis, Y. Paiss, E. Moshe, A. Ludmirsky, M. Werdiger, B. Arad, and A. Zigler, Phys. Lett. A 246, 329 (1998); L. M. Gorbunov and R. R. Ramazashvili,
Zh. Eksp.Teor. Fiz. 114, 849 (1998) [Sov. Phys. JETP 87, 461 (1998)].

[40] D. B. Milošević and A. F. Starace, Phys. Rev. Lett. 82, 2653 (1999).

[41] For the $\mathrm{H}$ atom ground state this is certainly valid, while for the $\mathrm{H}^{-}$negative ion ground state this approximation follows from results of C.-H. Park and A. F. Starace [Phys. Rev. A 29, 442 (1984)]. They showed that even for $B=9000 \mathrm{~T}$, the ground state wave function of $\mathrm{H}^{-}$is essentially unchanged for hyperspherical radii $R=\sqrt{r_{1}^{2}+r_{2}^{2}} \leqslant 6$, within which its peak amplitude is located. Only the tail of the wave function for $R$ $>6$ is changed slightly owing to the magnetic field compression.

[42] L. D. Landau and E. M. Lifshitz, Quantum Mechanics, 2nd ed. (Pergamon, Oxford, 1965).

[43] D. B. Milošević and A. F. Starace, Phys. Rev. Lett. 81, 5097 (1998).

[44] H. C. Bryant et al., Phys. Rev. A 27, 2889 (1983); S. Cohen et al., ibid. 36, 4728 (1987); W. W. Smith, et al. in Atomic Excitation and Recombination in External Fields, edited by M. H. Nayfeh and C. W. Clark (Gordon \& Breach, New York, 1985), p. 211; P. B. Keating et al., Phys. Rev. A 52, 4547 (1995).

[45] W. Manheimer, in The Physics of Particle Accelerators, edited by M. Month and M. Dienes, AIP Conf. Proc. No. 249 (AIP, New York, 1992), Vol. 2, p. 1794.

[46] Classically speaking, the electron rotates. Quantum mechanically, the electron wave function "breathes" in the plane perpendicular to the $B$-field axis.

[47] R. Loudon, The Quantum Theory of Light (Clarendon, Oxford, 1983).

[48] I. S. Gradshteyn and I. M. Ryzhik, Tables of Integrals, Series and Products (Academic, New York, 1965).

[49] Handbook of Mathematical Functions, edited by M. Abramowitz and I. A. Stegun (Dower, New York, 1965).

[50] D. E. Amos, ACM Trans. Math. Softw. 16, 169 (1990). 